\title{
A Stochastic Collocation Method for Elliptic Partial Differential Equations with Random Input Data*
}

\author{
Ivo Babuška ${ }^{\dagger}$ \\ Fabio Nobile $\ddagger$ \\ Raúl Tempone ${ }^{\S}$
}

\begin{abstract}
This work proposes and analyzes a stochastic collocation method for solving elliptic partial differential equations with random coefficients and forcing terms. These input data are assumed to depend on a finite number of random variables. The method consists of a Galerkin approximation in space and a collocation in the zeros of suitable tensor product orthogonal polynomials (Gauss points) in the probability space, and naturally leads to the solution of uncoupled deterministic problems as in the Monte Carlo approach. It treats easily a wide range of situations, such as input data that depend nonlinearly on the random variables, diffusivity coefficients with unbounded second moments, and random variables that are correlated or even unbounded. We provide a rigorous convergence analysis and demonstrate exponential convergence of the "probability error" with respect to the number of Gauss points in each direction of the probability space, under some regularity assumptions on the random input data. Numerical examples show the effectiveness of the method. Finally, we include a section with developments posterior to the original publication of this work. There we review sparse grid stochastic collocation methods, which are effective collocation strategies for problems that depend on a moderately large number of random variables.
\end{abstract}

Key words. stochastic collocation method, partial differential equations with random inputs, finite elements, uncertainty quantification, convergence rates, multivariate polynomial approximation, Smolyak approximation, anisotropic sparse approximation

AMS subject classifications. $65 \mathrm{~N} 35,65 \mathrm{~N} 15,65 \mathrm{C} 20,65 \mathrm{~N} 12,65 \mathrm{~N} 30$

DOI. $10.1137 / 100786356$

*Published electronically May 6, 2010. This paper originally appeared in SIAM Journal on Numerical Analysis, Volume 45, Number 3, 2007, pages 1005-1034.

http://www.siam.org/journals/sirev/52-2/78635.html

${ }^{\dagger}$ ICES, The University of Texas at Austin, Austin, TX 78712 (babuska@ices.utexas.edu). This author's work was partially supported by the Department of Energy (National Nuclear Security Administration) under award DE-FC52-08NA28615.

${ }_{\ddagger}$ MOX, Dipartimento di Matematica, Politecnico di Milano, 20133 Milano, Italy (fabio.nobile@ polimi.it). This author's work was partially supported by the Italian grants FIRB-IDEAS ("Advanced Numerical Techniques for Uncertainty Quantification in Engineering and Life Science Problems") and PRIN 2007 ("Modellistica numerica per il calcolo scientifico ed applicazioni avanzate").

$\S$ Applied Mathematics and Computational Sciences 4700, King Abdullah University of Science and Technology, Thuwal 23955-6900, Kingdom of Saudi Arabia (raul.tempone@kaust.edu.sa). This author's work was partially supported by the PECOS center at ICES, University of Texas at Austin (project 024550, Center for Predictive Computational Science), by the VR project "Effektiva numeriska metoder för stokastiska differentialekvationer med tillämpningar," and by King Abdullah University of Science and Technology (KAUST). 
Introduction. Thanks to the rapidly growing power of computers, numerical simulations are increasingly used to produce predictions of the behavior of complex physical or engineering systems. Some sources of errors arising in computer simulations now can be controlled and reduced by using sophisticated techniques such as a posteriori error estimation [1, 3, 57], mesh adaptivity, and the more recent modeling error analysis $[49,50,13]$. All this has increased the accuracy of numerical predictions as well as our confidence in them.

Yet many engineering applications are affected by a relatively large amount of uncertainty in the input data, such as model coefficients, forcing terms, boundary conditions, and geometry. In this case, to obtain a reliable numerical prediction, one has to include uncertainty quantification due to the uncertainty in the input data.

Uncertainty can be described in several ways, depending on the amount of information available; among others, we mention the worst-case scenario analysis, fuzzy set theory, evidence theory, and probabilistic setting. See [6, 35] and the references therein. In this paper we focus on elliptic partial differential equations (PDEs) with a probabilistic description of the uncertainty in the input data. The model problem has the form

$$
\mathcal{L}(a) u=f \quad \text { in } D
$$

where $\mathcal{L}$ is an elliptic operator in a domain $D \subset \mathbb{R}^{d}$, which depends on some coefficients $a(x, \omega)$, with $x \in D, \omega \in \Omega$, and $\Omega$ indicating the set of possible outcomes. Similarly, the forcing term $f=f(x, \omega)$ can be assumed to be random as well.

The first step in developing a stochastic collocation method consists in parametrizing the input randomness by means of a finite number of random variables. This can be the case if, for instance, the mathematical model depends on few parameters, which can be taken as random variables with a given joint probability distribution. For example, we might think of the deformation of an elastic homogeneous material in which Young's modulus and Poisson's ratio (parameters that characterize the material properties) are random variables, either independent or not.

In other situations, the input data may vary randomly from one point of the physical domain $D$ to another, and their uncertainty should rather be described in terms of random fields with a given covariance structure: the value at each point of the domain is a random variable, and the correlation between two distinct point values in the domain is known and in general is nonzero; this case is sometimes referred to as colored noise.

Examples of this situation are the deformation of inhomogeneous materials such as wood, foams, or biomaterials such as arteries and bones; groundwater flow problems where the permeability in each layer of sediment (rock, sand, etc.) should not be assumed constant; and the action of wind (direction and point intensity) on structures.

Although such random fields are infinite-dimensional, in general, several techniques are available to parametrize them with a given accuracy, by a finite number of random variables. We mention, for instance, the so-called Karhunen-Loève [39, 40] or a polynomial chaos (PC) expansion [59, 63]. The former represents the random field as a linear combination of an infinite number of uncorrelated random variables, while the latter uses polynomial expansions in terms of independent random variables. Both expansions exist, provided that the random field $a: \Omega \rightarrow V$, as a mapping from the probability space into a functional space $V$, has bounded second moments. Other nonlinear expansions can be considered as well (see, e.g., [33] for a technique to express a stationary random field with given covariance structure and marginal distribution 
as a function of countably many independent random variables; nonlinear transformations also have been used in $[43,60])$. The use of nonpolynomial expansions may be advantageous in some situations: for instance, in groundwater flow problems, the permeability coefficient within each layer of sediment can feature huge variability, which is often expressed in a logarithm scale. In this case, one might want to use a Karhunen-Loève (or PC) expansion for the logarithm of the permeability, instead of the permeability field itself. This leads to an exponential dependence of the permeability on the random variables, and the resulting random field might even have unbounded second moments. An advantage of such a nonlinear expansion is that it guarantees a positive permeability almost surely (a condition which is difficult to enforce with a standard truncated Karhunen-Loève or PC expansion).

To solve numerically the stochastic PDE (0.1), a numerical technique which has gained much attention in the last few years is the so-called stochastic Galerkin approximation (see, e.g., [32]). It employs standard approximations in space (finite elements, finite volumes, spectral or h-p finite elements, etc.) and polynomial approximation in the probability domain, either by total degree polynomial spaces [62, 43, 31, 52], tensor product polynomial spaces [4, 27], or piecewise polynomial spaces [4, 37].

The use of tensor product spaces is particularly attractive in the case of a small number of random variables, since it allows naturally the use of anisotropic spaces where the polynomial degree is chosen differently with respect to each random variable.

On the other hand, tensor product spaces suffer from the so-called curse of dimensionality since the dimension of the approximating space grows exponentially fast in the number of random variables. If the number of random variables is moderate or large, one should rather consider total degree polynomial spaces or sparse tensor product spaces [9, 27, 61]. The original publication of this work [7] did not address this case, but our subsequent works did. For instance, section 6 reviews sparse grid stochastic collocation methods, which are effective collocation strategies for problems that depend on a moderately large number of random variables.

The extension of the spectral Galerkin method to cases in which the input data depend nonlinearly on the random variables and possibly have unbounded second moments is not straightforward and, in any case, would lead to fully coupled systems of equations, which call for highly efficient parallel solvers.

In this work we propose a collocation method which consists in collocating problem (0.1) in the zeros of tensor product orthogonal polynomials with respect to the joint probability density $\rho$ of the random variables, should they be independent, or any other auxiliary density $\hat{\rho}$ corresponding to independent random variables, as long as the ratio $\rho / \hat{\rho}$ is bounded. Stochastic collocation has already been applied in a variety of problems and is the subject of ongoing research; see, among others, [54, 42] and the recent work [61], which the authors became aware of upon completion of this work.

As will be pointed out in the paper, this method offers several advantages:

- It naturally leads to uncoupled deterministic problems also in the case of input data which depend nonlinearly on the random variables.

- It treats efficiently the case of nonindependent random variables with the introduction of the auxiliary density $\hat{\rho}$.

- It can easily deal with unbounded random variables, such as Gaussian or exponential ones.

- It deals without difficulty with a diffusivity coefficient $a$ with unbounded second moment. 
The main result of the paper is given in Theorem 4.1 in section 4, where we prove that the collocation method preserves the same accuracy as the stochastic Galerkin approach and achieves exponential convergence in all the above-mentioned cases, provided that the input data are infinitely differentiable with respect to the random variables, under very mild assumptions on the growth of such derivatives, as is the case for standard expansions of random fields.

The collocation method can also be seen as a pseudospectral method (see, e.g., [51] and [28] for unbounded domains), i.e., a stochastic Galerkin approximation with the use of suitable Gaussian quadrature formulas. We will also show that in some particular cases, where such Gaussian quadratures are exact, it actually coincides with the stochastic Galerkin method based on tensor product spaces.

The outline of the paper is as follows: In section 1 we introduce the mathematical problem and the main notation used throughout. In section 2 we describe the collocation method. In section 3 we provide some regularity results on the solution of the stochastic PDE. In particular, we show that the solution is analytic with respect to the random variables, provided that the input data, as functions of the random variables, have infinite derivatives which do not grow too fast. In section 4 we give a complete convergence result for the collocation method and prove exponential convergence. Then, in section 5 , we present some numerical results showing the effectiveness of the proposed method. Finally, section 6 reviews sparse grid stochastic collocation methods, which are effective collocation strategies for problems that depend on a moderately large number of random variables. This last section presents sparse collocation methods, some results on convergence rates, and two numerical examples, including a numerical comparison with stochastic Galerkin.

I. Problem Setting. Let $D$ be a convex bounded polygonal domain in $\mathbb{R}^{d}$, and let $(\Omega, \mathcal{F}, P)$ be a complete probability space. Here $\Omega$ is the set of outcomes, $\mathcal{F} \subset 2^{\Omega}$ is the $\sigma$-algebra of events, and $P: \mathcal{F} \rightarrow[0,1]$ is a probability measure. Consider the stochastic linear elliptic boundary value problem: find a random function, $u$ : $\Omega \times \bar{D} \rightarrow \mathbb{R}$, such that $P$-almost everywhere (a.e.) in $\Omega$, or in other words, almost surely (a.s.) the following equation holds:

$$
\begin{gathered}
-\nabla \cdot(a(\omega, \cdot) \nabla u(\omega, \cdot))=f(\omega, \cdot) \text { on } D, \\
u(\omega, \cdot)=0 \text { on } \partial D .
\end{gathered}
$$

We will make the following assumptions:

$\left(\mathrm{A}_{1}\right) a(\omega, \cdot)$ is uniformly bounded from below; i.e.,

$$
\text { there exist } a_{\min }>0 \text { such that } P\left(\omega \in \Omega: a(\omega, x)>a_{\min } \forall x \in \bar{D}\right)=1 \text {. }
$$

$\left(\mathrm{A}_{2}\right) f(\omega, \cdot)$ is square integrable with respect to $P$; i.e., $\int_{D} E\left[f^{2}\right] d x<\infty$.

Moreover, we introduce the following Hilbert spaces:

- $V_{P}=L_{P}^{2}(\Omega) \otimes H_{0}^{1}(D)$, equipped with the norm $\|v\|_{P}^{2}=\int_{D} E\left[|\nabla v|^{2}\right] d x$.

- $V_{P, a} \equiv\left\{v \in V_{P}: \int_{D} E\left[a|\nabla v|^{2}\right] d x<\infty\right\}$, equipped with the norm $\|v\|_{P, a}=$ $\sqrt{\int_{D} E\left[a|\nabla v|^{2}\right] d x}$.

Observe that under the above assumptions, the space $V_{P, a}$ is continuously embedded in $V_{P}$ and

$$
\|v\|_{P} \leq \frac{1}{\sqrt{a_{\min }}}\|v\|_{P, a}
$$

Copyright $@$ by SIAM. Unauthorized reproduction of this article is prohibited. 
Problem (1.1) can be written in the following weak form:

$$
\text { find } u \in V_{P, a} \text { such that } \int_{D} E[a \nabla u \cdot \nabla v] d x=\int_{D} E[f v] d x \quad \forall v \in V_{P, a} .
$$

A straightforward application of the Lax-Milgram theorem allows one to state the well-posedness of problem (1.2) in the following lemma.

Lemma 1.1. Under assumptions $\left(\mathrm{A}_{1}\right)$ and $\left(\mathrm{A}_{2}\right)$, problem (1.2) admits a unique solution $u \in V_{P, a}$ which satisfies the estimate

$$
\|u\|_{P} \leq \frac{C_{P}}{a_{\min }}\left(\int_{D} E\left[f^{2}\right] d x\right)^{\frac{1}{2}}
$$

In the previous lemma we have used the Poincaré inequality

$$
\|w\|_{L^{2}(D)} \leq C_{P}\|\nabla w\|_{L^{2}(D)} \quad \forall w \in H_{0}^{1}(D) .
$$

Weaker Assumptions on the Random Coefficients. It is possible to relax the assumptions $\left(\mathrm{A}_{1}\right)$ and $\left(\mathrm{A}_{2}\right)$ substantially and still guarantee the existence and uniqueness of the solution $u$ to problem (1.2). In particular, if the lower bound for the coefficient $a$ is no longer a constant but a random variable, i.e., $a(x, \omega) \geq a_{\min }(\omega)>0$ a.s. a.e. on $D$, we have the following estimate for the moments of the solution.

Lemma 1.2 (moments estimates). Let $p, q \geq 0$ with $1 / p+1 / q=1$. Take a positive integer $k$. Then if $f \in L_{P}^{k p}\left(\Omega ; L^{2}(D)\right)$ and $1 / a_{\text {min }} \in L_{P}^{k q}(\Omega)$, we have that $u \in L_{P}^{k}\left(\Omega ; H_{0}^{1}(D)\right)$.

Proof. Since

$$
\|u(\cdot, \omega)\|_{H_{0}^{1}(D)} \leq C_{P} \frac{\|f(\cdot, \omega)\|_{L^{2}(D)}}{a_{\min }(\omega)} \text { a.s. }
$$

the result is a direct application of Hölder's inequality:

$$
\begin{aligned}
& \int_{\Omega}\|u(\cdot, \omega)\|_{H_{0}^{1}(D)}^{k} d P(\omega) \leq C_{P}^{k} \int_{\Omega}\left(\frac{\|f(\cdot, \omega)\|_{L^{2}(D)}}{a_{\min }(\omega)}\right)^{k} d P(\omega) \\
& \leq C_{P}^{k}\left(\int_{\Omega}\|f(\cdot, \omega)\|_{L^{2}(D)}^{k p} d P(\omega)\right)^{1 / p}\left(\int_{\Omega}\left(\frac{1}{a_{\min }(\omega)}\right)^{q k} d P(\omega)\right)^{1 / q} .
\end{aligned}
$$

Example 1 (lognormal diffusion coefficient). As an application of the previous lemma, we can conclude the well-posedness of (1.2) with a lognormal diffusion coefficient. For instance, let

$$
a(x, \omega)=\exp \left(\sum_{n=1}^{N} b_{n}(x) Y_{n}(\omega)\right), \quad Y_{n} \sim N(0,1) \quad \begin{aligned}
& \text { independent and identically } \\
& \text { distributed }
\end{aligned}
$$

Use the lower bound

$$
a_{\min }(\omega)=\exp \left(-\sum_{n=1}^{N}\left\|b_{n}\right\|_{L^{\infty}(D)}\left|Y_{n}(\omega)\right|\right),
$$

Copyright (c) by SIAM. Unauthorized reproduction of this article is prohibited. 
and then for $k, q<\infty$

$$
\begin{aligned}
& \left\|1 / a_{\min }\right\|_{L_{P}^{k q}(\Omega)}^{k q}=\int_{\Omega}\left(\frac{1}{a_{\min }(\omega)}\right)^{q k} d P(\omega) \\
& \quad=\int_{\mathbb{R}^{N}} \exp \left(k q \sum_{n=1}^{N}\left\|b_{n}\right\|_{L^{\infty}(D)}\left|z_{n}\right|\right) \exp \left(-\frac{1}{2} \sum_{n=1}^{N} z_{n}^{2}\right) d z_{1} \cdots d z_{N}<\infty .
\end{aligned}
$$

Now let $\epsilon>0$. Then by Lemma 1.2 the assumption $f \in L_{P}^{k(1+\epsilon)}\left(\Omega ; L^{2}(D)\right)$ together with (1.4) implies $u \in L_{P}^{k}\left(\Omega ; H_{0}^{1}(D)\right)$.

I.I. Finite-Dimensional Noise Assumption. In many problems the source of randomness can be approximated using just a small number of uncorrelated, sometimes independent, random variables; take, for example, the case of a truncated Karhunen-Loève expansion [39, 40]. This motivates us to make the following assumption.

Assumption 1 (finite-dimensional noise). The coefficients used in the computations have the form

$a(\omega, x)=a\left(Y_{1}(\omega), \ldots, Y_{N}(\omega), x\right)$ and $f(\omega, x)=f\left(Y_{1}(\omega), \ldots, Y_{N}(\omega), x\right)$ on $\Omega \times \bar{D}$, where $N \in \mathbb{N}_{+}$and $\left\{Y_{n}\right\}_{n=1}^{N}$ are real-valued random variables with mean value zero and unit variance.

We will denote with $\Gamma_{n} \equiv Y_{n}(\Omega)$ the image of $Y_{n}, \Gamma=\prod_{n=1}^{N} \Gamma_{n}$, and we will assume that the random variables $\left[Y_{1}, Y_{2}, \ldots, Y_{N}\right]$ have a joint probability density function $\rho: \Gamma \rightarrow \mathbb{R}_{+}$, with $\rho \in L^{\infty}(\Gamma)$.

Example 2. The following standard transformation guarantees that the diffusivity coefficient is bounded away from zero a.s.:

$$
\log \left(a-a_{\min }\right)(\omega, x)=b_{0}(x)+\sum_{1 \leq n \leq N} \sqrt{\lambda_{n}} b_{n}(x) Y_{n}(\omega)
$$

i.e., one performs a Karhunen-Loève expansion for $\log \left(a-a_{\min }\right)$, assuming that $a>a_{\min }$ a.s. On the other hand, the right-hand side of (1.1) can be represented as a truncated Karhunen-Loève expansion:

$$
f(\omega, x)=c_{0}(x)+\sum_{1 \leq n \leq N} \sqrt{\mu_{n}} c_{n}(x) Y_{n}(\omega)
$$

Remark 1. It is usual to have $f$ and $a$ independent, because the loads and the material properties are seldom related. In such a situation we have $a(Y(\omega), x)=$ $a\left(Y_{a}(\omega), x\right)$ and $f(Y(\omega), x)=f\left(Y_{f}(\omega), x\right)$, with $Y=\left[Y_{a}, Y_{f}\right]$ and the vectors $Y_{a}, Y_{f}$ independent.

After making Assumption 1, the solution $u$ of the stochastic elliptic boundary value problem (1.2) can be described by just a finite number of random variables, i.e., $u(\omega, x)=u\left(Y_{1}(\omega), \ldots, Y_{N}(\omega), x\right)$. Thus, the goal is to approximate the function $u=$ $u(y, x)$, where $y \in \Gamma$ and $x \in \bar{D}$. Observe that the stochastic variational formulation (1.2) has a "deterministic" equivalent which is the following: find $u \in V_{\rho, a}$ such that

$$
\int_{\Gamma} \rho(a \nabla u, \nabla v)_{L^{2}(D)} d y=\int_{\Gamma} \rho(f, v)_{L^{2}(D)} d y \quad \forall v \in V_{\rho, a},
$$

noting that here and later in this work the gradient notation, $\nabla$, always means differentiation with respect to $x \in D$ only, unless otherwise stated. The space $V_{\rho, a}$ is the analogue of $V_{P, a}$ with $(\Omega, \mathcal{F}, P)$ replaced by $\left(\Gamma, \mathcal{B}^{N}, \rho d y\right)$. 
Since the solution to (1.6) is unique and is also a solution to (1.2), it follows that the solution has necessarily the form $u(\omega, x)=u\left(Y_{1}(\omega), \ldots, Y_{N}(\omega), x\right)$. The stochastic boundary value problem (1.1) now becomes a deterministic Dirichlet boundary value problem for an elliptic PDE with an $N$-dimensional parameter. For convenience, we consider the solution $u$ as a function $u: \Gamma \rightarrow H_{0}^{1}(D)$ and use the notation $u(y)$ whenever we want to highlight the dependence on the parameter $y$. We use similar notation for the coefficient $a$ and the forcing term $f$. Then it can be shown that problem (1.1) is equivalent to

$$
\int_{D} a(y) \nabla u(y) \cdot \nabla \phi d x=\int_{D} f(y) \phi d x \quad \forall \phi \in H_{0}^{1}(D), \quad \rho \text {-a.e. in } \Gamma \text {. }
$$

For our convenience, we will suppose that the coefficient $a$ and the forcing term $f$ admit a smooth extension on the $\rho d y$-zero measure sets. Then (1.7) can be extended a.e. in $\Gamma$ with respect to the Lebesgue measure (instead of the measure $\rho d y$ ).

Remark 2. Strictly speaking, (1.7) will hold only for those values of $y \in \Gamma$ for which the coefficient $a(y)$ is finite. In this paper we will assume that $a(y)$ may go to infinity only at the boundary of the parameter domain $\Gamma$.

Making Assumption 1 is a crucial step, turning the original stochastic elliptic equation into a deterministic parametric elliptic equation and allowing the use of finite element and finite difference techniques to approximate the solution of the resulting deterministic problem (cf. [36, 20]).

Observe that the knowledge of $u=u(y, x)$ fully determines the law of the random field $u(\omega, x)$. However, the computation of some quantities of interest such as failure probabilities might pose extra challenges from the numerical point of view. On the other hand, computation of moments of the solution or functionals of the solution is direct (see sections 2 and 4.1).

2. Collocation Method. We seek a numerical approximation to the exact solution of (1.6) in a finite-dimensional subspace $V_{p, h}$ based on a tensor product, $V_{p, h}=\mathcal{P}_{p}(\Gamma) \otimes H_{h}(D)$, where the following hold:

- $H_{h}(D) \subset H_{0}^{1}(D)$ is a standard finite element space of dimension $N_{h}$, which contains continuous piecewise polynomials defined on regular triangulations $\mathcal{T}_{h}$ that have a maximum mesh spacing parameter $h>0$.

- $\mathcal{P}_{p}(\Gamma) \subset L_{\rho}^{2}(\Gamma)$ is the span of tensor product polynomials with degree at most $p=\left(p_{1}, \ldots, p_{N}\right) ;$ i.e., $\mathcal{P}_{p}(\Gamma)=\bigotimes_{n=1}^{N} \mathcal{P}_{p_{n}}\left(\Gamma_{n}\right)$, with

$$
\mathcal{P}_{p_{n}}\left(\Gamma_{n}\right)=\operatorname{span}\left(y_{n}^{m}, m=0, \ldots, p_{n}\right), \quad n=1, \ldots, N .
$$

Hence the dimension of $\mathcal{P}_{p}$ is $N_{p}=\prod_{n=1}^{N}\left(p_{n}+1\right)$.

We first introduce the semidiscrete approximation $u_{h}: \Gamma \rightarrow H_{h}(D)$, obtained by projecting (1.7) onto the subspace $H_{h}(D)$, for each $y \in \Gamma$, i.e.,

$$
\int_{D} a(y) \nabla u_{h}(y) \cdot \nabla \phi_{h} d x=\int_{D} f(y) \phi_{h} d x \quad \forall \phi_{h} \in H_{h}(D) \text { for a.e. } y \in \Gamma \text {. }
$$

The next step consists in collocating (2.1) on the zeros of orthogonal polynomials and building the discrete solution $u_{h, p} \in \mathcal{P}_{p}(\Gamma) \otimes H_{h}(D)$ by interpolating in $y$ the collocated solutions.

To this end, we first introduce an auxiliary probability density function $\hat{\rho}: \Gamma \rightarrow$ $\mathbb{R}^{+}$that can be seen as the joint probability of $N$ independent random variables; i.e., 
it factorizes as

$$
\hat{\rho}(y)=\prod_{n=1}^{N} \hat{\rho}_{n}\left(y_{n}\right) \forall y \in \Gamma \quad \text { and is such that }\left\|\frac{\rho}{\hat{\rho}}\right\|_{L^{\infty}(\Gamma)}<\infty .
$$

For each dimension $n=1, \ldots, N$, let $y_{n, k_{n}}, 1 \leq k_{n} \leq p_{n}+1$, be the $p_{n}+1$ roots of the orthogonal polynomial $q_{p_{n}+1}$ with respect to the weight $\hat{\rho}_{n}$, which then satisfies $\int_{\Gamma_{n}} q_{p_{n}+1}(y) v(y) \hat{\rho}_{n}(y) d y=0$ for all $v \in \mathcal{P}_{p_{n}}\left(\Gamma_{n}\right)$.

Standard choices for $\hat{\rho}$, such as constant, Gaussian, etc., lead to well-known roots of the polynomial $q_{p_{n}+1}$, which are tabulated to full accuracy and do not need to be computed.

To any vector of indexes $\left[k_{1}, \ldots, k_{N}\right]$ we associate the global index

$$
k=k_{1}+p_{1}\left(k_{2}-1\right)+p_{1} p_{2}\left(k_{3}-1\right)+\cdots,
$$

and we denote by $y_{k}$ the point $y_{k}=\left[y_{1, k_{1}}, y_{2, k_{2}}, \ldots, y_{N, k_{N}}\right] \in \Gamma$. We also introduce, for each $n=1,2, \ldots, N$, the Lagrange basis $\left\{l_{n, j}\right\}_{j=1}^{p_{n}+1}$ of the space $\mathcal{P}_{p_{n}}$,

$$
l_{n, j} \in \mathcal{P}_{p_{n}}\left(\Gamma_{n}\right), \quad l_{n, j}\left(y_{n, k}\right)=\delta_{j k}, \quad j, k=1, \ldots, p_{n}+1,
$$

where $\delta_{j k}$ is the Kronecker symbol, and we set $l_{k}(y)=\prod_{n=1}^{N} l_{n, k_{n}}\left(y_{n}\right)$. Hence, the final approximation is given by

$$
u_{h, p}(y, x)=\sum_{k=1}^{N_{p}} u_{h}\left(y_{k}, x\right) l_{k}(y)
$$

where $u_{h}\left(y_{k}, x\right)$ is the solution of problem (2.1) for $y=y_{k}$.

Equivalently, if we introduce the Lagrange interpolant operator $\mathcal{I}_{p}: C^{0}\left(\Gamma ; H_{0}^{1}(D)\right)$ $\rightarrow \mathcal{P}_{p}(\Gamma) \otimes H_{0}^{1}(D)$, such that

$$
\mathcal{I}_{p} v(y)=\sum_{k=1}^{N_{p}} v\left(y_{k}\right) l_{k}(y) \quad \forall v \in C^{0}\left(\Gamma ; H_{0}^{1}(D)\right),
$$

then we have simply $u_{h, p}=\mathcal{I}_{p} u_{h}$.

Finally, for any continuous function $g: \Gamma \rightarrow \mathbb{R}$ we introduce the Gauss quadrature formula $E_{\hat{\rho}}^{p}[g]$ approximating the integral $\int_{\Gamma} g(y) \hat{\rho}(y) d y$ as

$$
E_{\hat{\rho}}^{p}[g]=\sum_{k=1}^{N_{p}} \omega_{k} g\left(y_{k}\right), \quad \omega_{k}=\prod_{n=1}^{N} \omega_{k_{n}}, \quad \omega_{k_{n}}=\int_{\Gamma_{n}} l_{k_{n}}^{2}(y) \hat{\rho}_{n}(y) d y .
$$

This can be used to approximate the mean value or the variance of $u$ as

$$
\begin{aligned}
& \bar{u}_{h} \in H_{h}(D), \quad \bar{u}_{h}(x)=E_{\hat{\rho}}^{p}\left[\frac{\rho}{\hat{\rho}} u_{h}(x)\right], \\
& \operatorname{var}_{h}\left(u_{h}\right) \in L^{1}(D), \quad \operatorname{var}_{h}\left(u_{h}\right)(x)=E_{\hat{\rho}}^{p}\left[\frac{\rho}{\hat{\rho}}\left(u_{h}(x)-\bar{u}_{h}(x)\right)^{2}\right]
\end{aligned}
$$

as long as $\rho / \hat{\rho}$ is a smooth function. Otherwise, $\bar{u}_{h}$ and $\operatorname{var}_{h}\left(u_{h}\right)$ should be computed with a suitable quadrature formula which takes into account eventual discontinuities or singularities of $\rho / \hat{\rho}$.

Copyright (c) by SIAM. Unauthorized reproduction of this article is prohibited. 
2.I. Collocation versus Stochastic Galerkin Approximation. An alternative approach to the collocation method introduced thus far consists in approximating problem (1.6) with a stochastic Galerkin method: find $u_{h, p}^{G} \in \mathcal{P}_{p}(\Gamma) \otimes H_{h}(D)$ such that

$$
\int_{\Gamma} \rho\left(a \nabla u_{h, p}^{G}, \nabla v\right)_{L^{2}(D)} d y=\int_{\Gamma} \rho(f, v)_{L^{2}(D)} d y \quad \forall v \in \mathcal{P}_{p}(\Gamma) \otimes H_{h}(D) .
$$

This approach has been considered by several authors [4, 20, 27, 62, 32, 43]. Observe that, in general, problem (2.4) leads to a fully coupled system of linear equations, whose dimension is $N_{h} \times N_{p}$ and that demands highly efficient strategies and parallel computations for its numerical solution [23]. Conversely, the collocation method requires only the solutions of $N_{p}$ uncoupled linear systems of dimension $N_{h}$ and is fully parallelizable.

In $[4,5]$ a particular choice of basis functions (named double orthogonal polynomials) for the space $P_{p}(\Gamma)$ is proposed. This choice allows us to decouple the system in the special case where the diffusivity coefficient and the forcing term are multilinear combinations of the random variables $Y_{n}(\omega)$ (as is the case if one performs a truncated linear Karhunen-Loève expansion) and the random variables are independent, i.e., $\rho(y)=\prod_{n=1}^{N} \rho_{n}\left(y_{n}\right)$. The proposed basis is then obtained by solving the following eigenvalue problems for each $n=1, \ldots, N$ :

$$
\int_{\Gamma_{n}} z \psi_{k n}(z) v(z) \rho_{n}(z) d z=c_{k n} \int_{\Gamma_{n}} \psi_{k n}(z) v(z) \rho_{n}(z) d z, \quad k=1, \ldots, p_{n}+1 .
$$

The eigenvectors $\psi_{k n}$ are normalized so as to satisfy the property

$$
\int_{\Gamma_{n}} \psi_{k n}(z) \psi_{j n}(z) \rho_{n}(z) d z=\delta_{k j}, \quad \int_{\Gamma_{n}} z \psi_{k n}(z) \psi_{j n}(z) \rho_{n}(z) d z=c_{k n} \delta_{k j} .
$$

See $[4,5]$ for further details on the double orthogonal basis.

We now aim at analyzing the analogies between the collocation and the stochastic Galerkin methods. The collocation method can be seen as a pseudospectral Galerkin method (see, e.g., [51]), where the integrals over $\Gamma$ in (2.4) are replaced by the quadrature formula (2.3): find $u_{h, p} \in \mathcal{P}_{p}(\Gamma) \otimes H_{h}(D)$ such that

$$
E_{\hat{\rho}}^{p}\left[\frac{\rho}{\hat{\rho}}\left(a \nabla u_{h, p}, \nabla v\right)_{L^{2}(D)}\right]=E_{\hat{\rho}}^{p}\left[\frac{\rho}{\hat{\rho}}(f, v)_{L^{2}(D)}\right] \quad \forall v \in \mathcal{P}_{p}(\Gamma) \otimes H_{h}(D) .
$$

Indeed, by choosing in (2.5) the test functions of the form $v(y, x)=l_{k}(y) \phi(x)$, where $\phi(x) \in H_{h}(D)$ and $l_{k}(y)$ is the Lagrange basis function associated to the knot $y_{k}$, $k=1, \ldots, N_{p}$, one is led to solve a sequence of uncoupled problems of the form (2.1) collocated in the points $y_{k}$, which, ultimately, gives the same solution as the collocation method.

In the particular case where the diffusivity coefficient and the forcing term are multilinear combinations of the random variables $Y_{n}(\omega)$ and the random variables are independent, it turns out that the quadrature formula is exact if one chooses $\hat{\rho}=\rho$. In this case, the solution obtained by the collocation method actually coincides with the stochastic Galerkin one. This can be seen easily by observing that, with the above assumptions, the integrand in (2.4), i.e., $\left(a \nabla u_{h, p} \cdot \nabla v\right)$, is a polynomial at most of degree $2 p_{n}+1$ in the variable $y_{n}$ and the Gauss quadrature formula is exact for polynomials up to degree $2 p_{n}+1$ integrated against the weight $\rho$.

The collocation method is a natural generalization of the stochastic Galerkin approach and has the following advantages:

Copyright $@$ by SIAM. Unauthorized reproduction of this article is prohibited. 
- It decouples the system of linear equations in $Y$ also in the case where the diffusivity coefficient $a$ and the forcing term $f$ are nonlinear functions of the random variables $Y_{n}$.

- It treats efficiently the case of nonindependent random variables with the introduction of the auxiliary measure $\hat{\rho}$.

- It can easily deal with unbounded random variables (see Theorem 4.1 in section 4).

As will be shown in section 4, the collocation method preserves the same accuracy as the stochastic Galerkin approach and achieves exponential convergence if the coefficient $a$ and forcing term $f$ are infinitely differentiable with respect to the random variables $Y_{n}$, under very mild requirements on the growth of their derivatives in $Y$.

As a final remark, we show that the double orthogonal polynomials proposed in [4] coincide with the Lagrange basis $l_{k}(y)$, and the eigenvalues $c_{k n}$ are nothing but the Gauss knots of integration.

Lemma 2.1. Let $\Gamma \subset \mathbb{R}, \rho: \Gamma \rightarrow \mathbb{R}$ be a positive weight, and let $\left\{\psi_{k}\right\}_{k=1}^{p+1}$ be the set of double orthogonal polynomials of degree $p$ satisfying

$$
\int_{\Gamma} \psi_{k}(y) \psi_{j}(y) \rho(y) d y=\delta_{k j}, \quad \int_{\Gamma} y \psi_{k}(y) \psi_{j}(y) \rho(y) d y=c_{k} \delta_{k j} .
$$

Then the eigenvalues $c_{k}$ are the nodes of the Gaussian quadrature formula associated to the weight $\rho$, and the eigenfunctions $\psi_{k}$ are, up to multiplicative factors, the corresponding Lagrange polynomials built on the nodes $c_{k}$.

Proof. We have, for $k=1, \ldots, p+1$,

$$
\int_{\Gamma}\left(y-c_{k}\right) \psi_{k}(y) v(y) \rho(y) d y=0 \quad \forall v \in P_{p}(\Gamma) .
$$

Take $v=\prod_{\substack{j=1 \\ j \neq k}}^{p+1}\left(y-c_{j}\right) \in P_{p}(\Gamma)$ in the above and let $w=\prod_{j=1}^{p+1}\left(y-c_{j}\right)$. Then

$$
\int_{\Gamma} w(y) \psi_{k}(y) \rho(y) d y=0 \quad \forall k=1, \ldots, p+1 .
$$

Since $\left\{\psi_{k}\right\}_{k=1}^{p+1}$ defines a basis of the space $\mathcal{P}_{p}(\Gamma)$, the previous relation implies that $w$ is $\rho$-orthogonal to $P_{p}(\Gamma)$. In addition, the functions $\left(y-c_{k}\right) \psi_{k}$ are also orthogonal to the same subspace: this yields, due to the one-dimensional nature of the orthogonal complement of $P_{p}(\Gamma)$ over $P_{p+1}(\Gamma)$,

$$
\left(y-c_{k}\right) \psi_{k}=\alpha_{k} w=\alpha_{k} \prod_{j=1}^{p+1}\left(y-c_{j}\right), \quad k=1, \ldots, p+1,
$$

which gives

$$
\psi_{k}=\alpha_{k} \prod_{\substack{j=1 \\ j \neq k}}^{p+1}\left(y-c_{j}\right), \quad k=1, \ldots, p+1
$$

i.e., the double orthogonal polynomials $\psi_{k}$ are collinear to Lagrange interpolants at the nodes $c_{j}$. Moreover, the eigenvalues $c_{j}$ are the roots of the polynomial $w \in P_{p+1}(\Gamma)$, which is $\rho$-orthogonal to $P_{p}(\Gamma)$, and therefore they coincide with the nodes of the Gaussian quadrature formula associated to the weight $\rho$. 
3. Regularity Results. Before going through the convergence analysis of the method, we need to state some regularity assumptions on the data of the problem and consequent regularity results for the exact solution $u$ and the semidiscrete solution $u_{h}$.

In what follows we will need some restrictive assumptions on $f$ and $\rho$. In particular, we will assume $f$ to be a continuous function in $y$, whose growth at infinity, whenever the domain $\Gamma$ is unbounded, is at most exponential. At the same time we will assume that $\rho$ behaves as a Gaussian weight at infinity, as does the auxiliary density $\hat{\rho}$, in light of assumption (2.2).

Other types of growth of $f$ at infinity and corresponding decay of the probability density $\rho$, for instance, of exponential type, could be considered as well. Yet we will limit the analysis to the aforementioned case.

To make these assumptions precise, we introduce a weight $\sigma(y)=\prod_{n=1}^{N} \sigma_{n}\left(y_{n}\right) \leq$ 1 , where

$$
\sigma_{n}\left(y_{n}\right)= \begin{cases}1 & \text { if } \Gamma_{n} \text { is bounded } \\ e^{-\alpha_{n}\left|y_{n}\right|} \text { for some } \alpha_{n}>0 & \text { if } \Gamma_{n} \text { is unbounded }\end{cases}
$$

and the functional space

$$
C_{\sigma}^{0}(\Gamma ; V) \equiv\left\{v: \Gamma \rightarrow V, \quad v \text { continuous in } y, \max _{y \in \Gamma}\|\sigma(y) v(y)\|_{V}<+\infty\right\},
$$

where $V$ is a Banach space of functions defined in $D$.

Assumption 2 (growth at infinity). In what follows we will assume that

(a) $f \in C_{\sigma}^{0}\left(\Gamma ; L^{2}(D)\right)$, and

(b) the joint probability density $\rho$ satisfies

$$
\rho(y) \leq C_{\rho} e^{-\sum_{n=1}^{N}\left(\delta_{n} y_{n}\right)^{2}} \quad \forall y \in \Gamma
$$

for some constant $C_{\rho}>0$ and $\delta_{n}$ strictly positive if $\Gamma_{n}$ is unbounded and zero otherwise.

The parameter $\delta_{n}$ in (3.2) gives a scale for the decay of $\rho$ at infinity and provides an estimate of the dispersion of the random variable $Y_{n}$. On the other hand, the parameter $\alpha_{n}$ in (3.1) controls the growth of the forcing term $f$ at infinity.

Remark 3 (growth of $f$ ). The convergence result given in Theorem 4.1 in section 4 extends to a wider class of functions $f$. For instance, we could take $f \in C_{\sigma}^{0}\left(\Gamma ; L^{2}(D)\right)$ with $\sigma=e^{-\sum_{n=1}^{N}\left(\delta_{n} y_{n}\right)^{2} / 8}$. Yet the class given in (3.1) is already large enough for most practical applications (see Example 2).

We can now choose any suitable auxiliary density $\hat{\rho}(y)=\prod_{n=1}^{N} \hat{\rho}_{n}\left(y_{n}\right)$ that satisfies, for each $n=1, \ldots, N$,

$$
C_{\min }^{n} e^{-\left(\delta_{n} y_{n}\right)^{2}} \leq \hat{\rho}_{n}\left(y_{n}\right)<C_{\max }^{n} e^{-\left(\delta_{n} y_{n}\right)^{2}} \quad \forall y_{n} \in \Gamma_{n}
$$

for some positive constants $C_{\min }^{n}$ and $C_{\max }^{n}$ that do not depend on $y_{n}$.

Observe that this choice satisfies the requirement given in (2.2), i.e., $\|\rho / \hat{\rho}\|_{L^{\infty}(\Gamma)} \leq$ $C_{\rho} / C_{\text {min }}$ with $C_{\text {min }}=\prod_{n=1}^{N} C_{m i n}^{n}$.

Under the above assumptions, the following inclusions hold true:

$$
C_{\sigma}^{0}(\Gamma ; V) \subset L_{\hat{\rho}}^{2}(\Gamma ; V) \subset L_{\rho}^{2}(\Gamma ; V)
$$

Copyright $@$ ㅇ by SIAM. Unauthorized reproduction of this article is prohibited. 
with continuous embedding. Indeed, on one hand we have

$$
\|v\|_{L_{\rho}^{2}(\Gamma ; V)} \leq\left\|\frac{\rho}{\hat{\rho}}\right\|_{L^{\infty}(\Gamma)}^{\frac{1}{2}}\|v\|_{L_{\hat{\rho}}^{2}(\Gamma ; V)} \leq \sqrt{\frac{C_{\rho}}{C_{\min }}}\|v\|_{L_{\hat{\rho}}^{2}(\Gamma ; V)} .
$$

On the other hand,

$$
\|v\|_{L_{\hat{\rho}}^{2}(\Gamma ; V)}^{2}=\int_{\Gamma} \hat{\rho}(y)\|v(y)\|_{V}^{2} d y \leq\|v\|_{C_{\sigma}^{0}(\Gamma ; V)}^{2} \int_{\Gamma} \frac{\hat{\rho}(y)}{\sigma^{2}(y)} d y \leq \prod_{n=1}^{N} M_{n}\|v\|_{C_{\sigma}^{0}(\Gamma ; V)}^{2}
$$

with $M_{n}=\int_{\Gamma_{n}} \hat{\rho}_{n} / \sigma_{n}^{2}$. Now, for $\Gamma_{n}$ bounded, $M_{n} \leq C_{m a x}^{n}\left|\Gamma_{n}\right|$, whereas if $\Gamma_{n}$ is unbounded,

$$
M_{n}=\int_{\Gamma_{n}}\left(e^{-\frac{\left(\delta_{n} y\right)^{2}}{2}+2 \alpha_{n}|y|}\right) e^{\frac{\left(\delta_{n} y\right)^{2}}{2}} \hat{\rho}_{n}(y) d y \leq C_{\max }^{n} \sqrt{\frac{2 \pi}{\delta_{n}}} e^{2\left(\alpha_{n} / \delta_{n}\right)^{2}} .
$$

The first result we need is the following lemma.

Lemma 3.1. If $f \in C_{\sigma}^{0}\left(\Gamma ; L^{2}(D)\right)$ and $a \in C_{l o c}^{0}\left(\Gamma ; L^{\infty}(D)\right)$, uniformly bounded away from zero, then the solution to problem (1.7) satisfies $u \in C_{\sigma}^{0}\left(\Gamma ; H_{0}^{1}(D)\right)$.

The proof of this lemma is immediate. The next result concerns the analyticity of the solution $u$ whenever the diffusivity coefficient $a$ and the forcing term $f$ are infinitely differentiable with respect to $y$, under mild assumptions on the growth of their derivatives in $y$. We will perform a one-dimensional analysis in each direction $y_{n}, n=1, \ldots, N$. For this, we introduce the following notation:

$$
\Gamma_{n}^{*}=\prod_{\substack{j=1 \\ j \neq n}}^{N} \Gamma_{j}
$$

with $y_{n}^{*}$ denoting an arbitrary element of $\Gamma_{n}^{*}$. Similarly, we set

$$
\hat{\rho}_{n}^{*}=\prod_{\substack{j=1 \\ j \neq n}}^{N} \hat{\rho}_{j}
$$

and

$$
\sigma_{n}^{*}=\prod_{\substack{j=1 \\ j \neq n}}^{N} \sigma_{j}
$$

LEMma 3.2. Under the assumption that, for every $y=\left(y_{n}, y_{n}^{*}\right) \in \Gamma$, there exists $\gamma_{n}<+\infty$ such that

$$
\left\|\frac{\partial_{y_{n}}^{k} a(y)}{a(y)}\right\|_{L^{\infty}(D)} \leq \gamma_{n}^{k} k ! \quad \text { and } \quad \frac{\left\|\partial_{y_{n}}^{k} f(y)\right\|_{L^{2}(D)}}{1+\|f(y)\|_{L^{2}(D)}} \leq \gamma_{n}^{k} k !
$$

the solution $u\left(y_{n}, y_{n}^{*}, x\right)$ as a function of $y_{n}, u: \Gamma_{n} \rightarrow C_{\sigma_{n}^{*}}^{0}\left(\Gamma_{n}^{*} ; H_{0}^{1}(D)\right)$ admits an analytic extension $u\left(z, y_{n}^{*}, x\right), z \in \mathbb{C}$, in the region of the complex plane

$$
\Sigma\left(\Gamma_{n} ; \tau_{n}\right) \equiv\left\{z \in \mathbb{C}, \operatorname{dist}\left(z, \Gamma_{n}\right) \leq \tau_{n}\right\}
$$

Copyright (c) by SIAM. Unauthorized reproduction of this article is prohibited. 
with $0<\tau_{n}<1 /\left(2 \gamma_{n}\right)$. Moreover, for all $z \in \Sigma\left(\Gamma_{n} ; \tau_{n}\right)$,

$$
\left\|\sigma_{n}(\operatorname{Re} z) u(z)\right\|_{C_{\sigma_{n}^{*}}^{0}\left(\Gamma_{n}^{*} ; H_{0}^{1}(D)\right)} \leq \frac{C_{P} e^{\alpha_{n} \tau_{n}}}{a_{\min }\left(1-2 \tau_{n} \gamma_{n}\right)}\left(2\|f\|_{C_{\sigma}^{0}\left(\Gamma ; H_{0}^{1}(D)\right)}+1\right)
$$

with the constant $C_{P}$ as in (1.3).

Proof. In every point $y \in \Gamma$, the $k$ th derivative of $u$ with respect to $y_{n}$ satisfies the problem

$$
B\left(y ; \partial_{y_{n}}^{k} u, v\right)=-\sum_{l=1}^{k}\left(\begin{array}{c}
k \\
l
\end{array}\right) \partial_{y_{n}}^{l} B\left(y ; \partial_{y_{n}}^{k-l} u, v\right)+\left(\partial_{y_{n}}^{k} f, v\right) \quad \forall v \in H_{0}^{1}(D),
$$

where $B$ is the parametric bilinear form $B(y ; u, v)=\int_{D} a(y) \nabla u \cdot \nabla v d x$. Hence

$$
\begin{aligned}
\left\|\sqrt{a(y)} \nabla \partial_{y_{n}}^{k} u\right\|_{L^{2}(D)} \leq & \sum_{l=1}^{k}\left(\begin{array}{c}
k \\
l
\end{array}\right)\left\|\frac{\partial_{y_{n} a(y)}^{l}}{a(y)}\right\|_{L^{\infty}(D)}\left\|\sqrt{a(y)} \nabla \partial_{y_{n}}^{k-l} u\right\|_{L^{2}(D)} \\
& +\frac{C_{P}}{\sqrt{a_{\min }}}\left\|\partial_{y_{n}}^{k} f\right\|_{L^{2}(D)} .
\end{aligned}
$$

Setting $R_{k}=\left\|\sqrt{a(y)} \nabla \partial_{y_{n}}^{k} u\right\|_{L^{2}(D)} / k$ ! and using the bounds on the derivatives of $a$ and $f$, we obtain the recursive inequality

$$
R_{k} \leq \sum_{l=1}^{k} \gamma_{n}^{l} R_{k-l}+\frac{C_{P}}{\sqrt{a_{\min }}} \gamma_{n}^{k}\left(1+\|f\|_{L^{2}(D)}\right) .
$$

The generic term $R_{k}$ admits the bound

$$
R_{k} \leq \frac{1}{2}\left(2 \gamma_{n}\right)^{k}\left[R_{0}+\frac{C_{P}}{\sqrt{a_{\min }}}\left(1+\|f\|_{L^{2}(D)}\right)\right] .
$$

Observing that $R_{0}=\|\sqrt{a(y)} \nabla u(y)\|_{L^{2}(D)} \leq \frac{C_{P}}{\sqrt{a_{m i n}}}\|f(y)\|_{L^{2}(D)}$ and

$$
\frac{\left\|\nabla \partial_{y_{n}}^{k} u\right\|_{L^{2}(D)}}{k !} \leq \frac{R_{k}}{\sqrt{a_{\min }}}
$$

we get the final estimate on the growth of the derivatives of $u$,

$$
\frac{\left\|\nabla \partial_{y_{n}}^{k} u(y)\right\|_{L^{2}(D)}}{k !} \leq \frac{C_{P}}{a_{\min }}\left(2\|f(y)\|_{L^{2}(D)}+1\right)\left(2 \gamma_{n}\right)^{k} .
$$

We now define for every $y_{n} \in \Gamma_{n}$ the power series $u: \mathbb{C} \rightarrow C_{\sigma_{n}^{*}}^{0}\left(\Gamma_{n}^{*}, H_{0}^{1}(D)\right)$ as

$$
u\left(z, y_{n}^{*}, x\right)=\sum_{k=0}^{\infty} \frac{\left(z-y_{n}\right)^{k}}{k !} \partial_{y_{n}}^{k} u\left(y_{n}, y_{n}^{*}, x\right)
$$

Hence,

$$
\begin{gathered}
\sigma_{n}\left(y_{n}\right)\|u(z)\|_{C_{\sigma_{n}^{*}}^{0}\left(\Gamma_{n}^{*}, H_{0}^{1}(D)\right)} \leq \sum_{k=0}^{\infty} \frac{\left|z-y_{n}\right|^{k}}{k !} \sigma_{n}\left(y_{n}\right)\left\|\partial_{y_{n}}^{k} u\left(y_{n}\right)\right\|_{C_{\sigma_{n}^{*}}^{0}\left(\Gamma_{n}^{*} ; H_{0}^{1}(D)\right)} \\
\leq \frac{C_{P}}{a_{\min }} \max _{y_{n} \in \Gamma_{n}}\left\{\sigma_{n}\left(y_{n}\right)\left(2\left\|f\left(y_{n}\right)\right\|_{C_{\sigma_{n}^{*}}^{0}\left(\Gamma_{n}^{*} ; L^{2}(D)\right)}+1\right)\right\} \sum_{k=0}^{\infty}\left(\left|z-y_{n}\right| 2 \gamma_{n}\right)^{k} \\
\leq \frac{C_{P}}{a_{\min }}\left(2\|f\|_{C_{\sigma}^{0}\left(\Gamma ; L^{2}(D)\right)}+1\right) \sum_{k=0}^{\infty}\left(\left|z-y_{n}\right| 2 \gamma_{n}\right)^{k},
\end{gathered}
$$

Copyright $@$ by SIAM. Unauthorized reproduction of this article is prohibited. 
where we have exploited the fact that $\sigma_{n}\left(y_{n}\right) \leq 1$ for all $y_{n} \in \Gamma_{n}$; the series converges for all $z \in \mathbb{C}$ such that $\left|z-y_{n}\right| \leq \tau_{n}<1 /\left(2 \gamma_{n}\right)$. Moreover, in the ball $\left|z-y_{n}\right| \leq \tau_{n}$, we have, by virtue of (3.1), $\sigma_{n}(\operatorname{Re} z) \leq e^{\alpha_{n} \tau_{n}} \sigma_{n}\left(y_{n}\right)$, and then

$$
\sigma_{n}(\operatorname{Re} z)\|u(z)\|_{C_{\sigma_{n}^{*}}^{0}\left(\Gamma_{n}^{*}, H_{0}^{1}(D)\right)} \leq \frac{C_{P} e^{\alpha_{n} \tau_{n}}}{a_{\min }\left(1-2 \tau_{n} \gamma_{n}\right)}\left(2\|f\|_{C_{\sigma}^{0}\left(\Gamma ; L^{2}(D)\right)}+1\right) .
$$

The power series converges for every $y_{n} \in \Gamma_{n}$; hence, by a continuation argument, the function $u$ can be extended analytically on the whole region $\Sigma\left(\Gamma_{n} ; \tau_{n}\right)$, and estimate (3.6) follows.

Example 3. Let us consider the case where the diffusivity coefficient $a$ is expanded in a linear truncated Karhunen-Loève expansion

$$
a(\omega, x)=b_{0}(x)+\sum_{n=1}^{N} \sqrt{\lambda_{n}} b_{n}(x) Y_{n}(\omega),
$$

provided that such an expansion guarantees $a(\omega, x) \geq a_{\text {min }}$ for almost every $\omega \in \Omega$ and $x \in D[27]$. In this case we have

$$
\left\|\frac{\partial_{y_{n}}^{k} a}{a}\right\|_{L^{\infty}(\Gamma \times D)} \leq \begin{cases}\sqrt{\lambda_{n}}\left\|b_{n}\right\|_{L^{\infty}(D)} / a_{\min } & \text { for } k=1, \\ 0 & \text { for } k>1,\end{cases}
$$

and we can safely take $\gamma_{n}=\sqrt{\lambda_{n}}\left\|b_{n}\right\|_{L^{\infty}(D)} / a_{\min }$ in (3.4).

If we consider, instead, a truncated exponential expansion

$$
a(\omega, x)=a_{\min }+e^{b_{0}(x)+\sum_{n=1}^{N} \sqrt{\lambda_{n}} b_{n}(x) Y_{n}(\omega)},
$$

we have

$$
\left\|\frac{\partial_{y_{n}}^{k} a}{a}\right\|_{L^{\infty}(\Gamma \times D)} \leq\left(\sqrt{\lambda_{n}}\left\|b_{n}\right\|_{L^{\infty}(D)}\right)^{k},
$$

and we can take $\gamma_{n}=\sqrt{\lambda_{n}}\left\|b_{n}\right\|_{L^{\infty}(D)}$. Hence, both choices fulfill the assumption in Lemma 3.2 .

Example 4. Similarly to the previous case, let us consider a forcing term $f$ of the form

$$
f(\omega, x)=c_{0}(x)+\sum_{n=1}^{N} c_{n}(x) Y_{n}(\omega),
$$

where the random variables $Y_{n}$ are Gaussian (either independent or not) and the functions $c_{n}(x)$ are square integrable for any $n=1, \ldots, N$. Then the function $f$ belongs to the space $C_{\sigma}^{0}\left(\Gamma ; L^{2}(D)\right)$, with weight $\sigma$ defined in (3.1), for any choice of the exponent coefficients $\alpha_{n}>0$.

Moreover,

$$
\frac{\left\|\partial_{y_{n}}^{k} f(y)\right\|_{L^{2}(D)}}{1+\|f(y)\|_{L^{2}(D)}} \leq \begin{cases}\left\|c_{n}\right\|_{L^{2}(D)} & \text { for } k=1, \\ 0 & \text { for } k>1,\end{cases}
$$

and we can safely take $\gamma_{n}=\left\|c_{n}\right\|_{L^{2}(D)}$ in (3.4). Hence, such a forcing term satisfies the assumptions of Lemma 3.2. In this case, though, the solution $u$ is linear with respect to the random variables $Y_{n}$ (hence, clearly analytic), and our theory is not needed.

Observe that the regularity results are valid also for the semidiscrete solution $u_{h}$. 
4. Convergence Analysis. Our aim is to give a priori estimates for the total error $\epsilon=u-u_{h, p}$ in the natural norm $L_{\rho}^{2}(\Gamma) \otimes H_{0}^{1}(D)$. The next theorem states the convergence result we are seeking, and the rest of the section will be devoted to its proof. In particular, we will prove that the error decays (sub)exponentially fast with respect to $p$ under the regularity assumptions made in section 3. The convergence with respect to $h$ will be dictated by standard approximability properties of the finite element space $H_{h}(D)$ and the regularity in space of the solution $u$ (see, e.g., [18, 14]).

THEOREM 4.1. Under the assumptions of Lemmas 3.1 and 3.2, there exist positive constants $r_{n}, n=1, \ldots, N$, and $C$, independent of $h$ and $p$, such that

$$
\begin{array}{r}
\left\|u-u_{h, p}\right\|_{L_{\rho}^{2} \otimes H_{0}^{1}} \leq \frac{1}{\sqrt{a_{\min }}} \inf _{v \in L_{\rho}^{2} \otimes H_{h}}\left(\int_{\Gamma \times D} \rho a|\nabla(u-v)|^{2}\right)^{\frac{1}{2}} \\
+C \sum_{n=1}^{N} \beta_{n}\left(p_{n}\right) \exp \left\{-r_{n} p_{n}^{\theta_{n}}\right\},
\end{array}
$$

where

- if $\Gamma_{n}$ is bounded,

$$
\left\{\begin{array}{l}
\theta_{n}=\beta_{n}=1, \\
r_{n}=\log \left[\frac{2 \tau_{n}}{\left|\Gamma_{n}\right|}\left(1+\sqrt{1+\frac{\left|\Gamma_{n}\right|^{2}}{4 \tau_{n}^{2}}}\right)\right],
\end{array}\right.
$$

- if $\Gamma_{n}$ is unbounded,$$
\left\{\begin{array}{l}
\theta_{n}=1 / 2, \quad \beta_{n}=O\left(\sqrt{p_{n}}\right), \\
r_{n}=\tau_{n} \delta_{n},
\end{array}\right.
$$

$\tau_{n}$ is smaller than the distance between $\Gamma_{n}$ and the nearest singularity in the complex plane, as defined in Lemma 3.2, and $\delta_{n}$ is defined as in (3.2).

The first term on the right-hand side of (4.1) concerns the space approximability of $u$ in the subspace $H_{h}(D)$ and is controlled by the mesh size $h$. The actual rate of convergence will depend on the regularity in space of $a(y)$ and $f(y)$ for each $y \in \Gamma$ as well as on the smoothness on the domain $D$. Observe that an $h$ or $h-p$ adaptive strategy to reduce the error in space is not precluded by this approach.

The exponential rate of convergence in the $Y$ direction depends on the constants $r_{n}$, which in turn are related to the distances from the sets $\Gamma_{n}$ to their nearest singularities in the complex plane. In Examples 3 and 4 we have estimated these constants in the case where the random fields $a$ and $f$ are represented by either a linear or exponential truncated Karhunen-Loève expansion. Hence, a full characterization of the convergence rate is available in these cases.

Observe that in Theorem 4.1 it is not necessary to assume the finiteness of the second moment of the coefficient $a$.

Before proving the theorem, we recall some known results of approximation theory for a function $f$ defined on a one-dimensional domain (bounded or unbounded) with values in a Hilbert space $V, f: \Gamma \subset \mathbb{R} \rightarrow V$. As in section 2 , let $\rho: \Gamma \rightarrow \mathbb{R}^{+}$be a positive weight which satisfies, for all $y \in \Gamma, \rho(y) \leq C_{M} e^{-(\delta y)^{2}}$ for some $C_{M}>0$ and $\delta$ strictly positive if $\Gamma$ is unbounded and zero otherwise; let $y_{k} \in \Gamma, k=1, \ldots, p+1$, be the set of zeros of the polynomial of degree $p$ orthogonal to the space $\mathcal{P}_{p-1}$ with respect to the weight $\rho$; and let $\sigma$ be an extra positive weight such that $\sigma(y) \geq C_{m} e^{-(\delta y)^{2} / 4}$ for some $C_{m}>0$. With this choice, the embedding $C_{\sigma}^{0}(\Gamma ; V) \subset L_{\rho}^{2}(\Gamma ; V)$ is continuous. Observe that the condition on $\sigma$ is satisfied both by a Gaussian weight $\sigma=e^{-(\mu y)^{2}}$ 
with $\mu \leq \delta / 2$ and by an exponential weight $\sigma=e^{-\alpha|y|}$ for any $\alpha \geq 0$. Finally, we denote by $\mathcal{I}_{p}$ the Lagrange interpolant operator, $\mathcal{I}_{p} v(y)=\sum_{k=1}^{p+1} v\left(y_{k}\right) l_{k}(y)$ for every continuous function $v$, and by $\omega_{k}=\int_{\Gamma} l_{k}^{2}(y) \rho(y) d y$ the weights of the Gaussian quadrature formula built upon $\mathcal{I}_{p}$.

The following two lemmas are a slight generalization of a classical result by Erdös and Turán [25].

LEMMA 4.2. The operator $\mathcal{I}_{p}: C_{\sigma}^{0}(\Gamma ; V) \rightarrow L_{\rho}^{2}(\Gamma ; V)$ is continuous.

Proof. We have, indeed, that for any $v \in C_{\sigma}^{0}(\Gamma ; V)$

$$
\left\|\mathcal{I}_{p} v\right\|_{L_{\rho}^{2}(\Gamma ; V)}^{2}=\int_{\Gamma}\left\|\sum_{k=1}^{p+1} v\left(y_{k}\right) l_{k}(y)\right\|_{V}^{2} \rho(y) d y .
$$

Thanks to the orthogonality property $\int_{\Gamma} l_{j}(y) l_{k}(y) \rho(y) d y=\delta_{j k}$, we have

$$
\begin{aligned}
\left\|\mathcal{I}_{p} v\right\|_{L_{\rho}^{2}(\Gamma ; V)}^{2} & =\int_{\Gamma} \sum_{k=1}^{p+1}\left\|v\left(y_{k}\right)\right\|_{V}^{2} l_{k}^{2}(y) \rho(y) d y \\
& \leq \max _{k=1, \ldots, p+1}\left\|v\left(y_{k}\right)\right\|_{V}^{2} \sigma^{2}\left(y_{k}\right) \sum_{k=1}^{p+1} \int_{\Gamma} \frac{l_{k}^{2}(y) \rho(y)}{\sigma^{2}\left(y_{k}\right)} d y \\
& \leq\|v\|_{C_{\sigma}^{0}(\Gamma ; V)}^{2} \sum_{k=1}^{p+1} \frac{\omega_{k}}{\sigma^{2}\left(y_{k}\right)} .
\end{aligned}
$$

In the case of $\Gamma$ bounded, we have $\sigma \geq C_{m}$ and $\sum_{k=1}^{p+1} \omega_{k}=1$ for any $p$, and the result follows immediately. For $\Gamma$ unbounded, since $\rho(y) \leq C_{M} e^{-(\delta y)^{2}}$, all the even moments $c_{2 m}=\int_{\Gamma} y^{2 m} \rho(y) d y$ are bounded, up to a constant, by the moments of the Gaussian density $e^{-(\delta y)^{2}}$. Therefore, using a result from Uspensky in 1928 [56], it follows that

$$
\sum_{k=1}^{p+1} \frac{\omega_{k}}{\sigma^{2}\left(y_{k}\right)} \stackrel{p \rightarrow \infty}{\longrightarrow} \int_{\Gamma} \frac{\rho(y)}{\sigma^{2}(y)} d y \leq \frac{C_{M}}{C_{m}^{2}} \sqrt{\frac{2 \pi}{\delta}},
$$

and we conclude that

$$
\left\|\mathcal{I}_{p} v\right\|_{L_{\rho}^{2}(\Gamma ; V)} \leq C_{1}\|v\|_{C_{\sigma}^{0}(\Gamma ; V)} .
$$

Observe that, in general, if $V$ is just Banach and not Hilbert, we obtain, for the worst case, $\left\|\mathcal{I}_{p} v\right\|_{L_{\rho}^{2}(\Gamma ; V)} \leq C_{1} \sqrt{p+1}\|v\|_{C_{\sigma}^{0}(\Gamma ; V)}$. Note that for specific choices of $\rho$ this estimate may be improved.

Lemma 4.3. For every function $v \in C_{\sigma}^{0}(\Gamma ; V)$ the interpolation error satisfies

$$
\left\|v-\mathcal{I}_{p} v\right\|_{L_{\rho}^{2}(\Gamma ; V)} \leq C_{2} \inf _{w \in \mathcal{P}_{p}(\Gamma) \otimes V}\|v-w\|_{C_{\sigma}^{0}(\Gamma ; V)}
$$

with a constant $C_{2}$ independent of $p$.

Proof. Let us observe that for all $w \in \mathcal{P}_{p}(\Gamma) \otimes V$, it holds that $\mathcal{I}_{p} w=w$. Then

$$
\begin{aligned}
\left\|v-\mathcal{I}_{p} v\right\|_{L_{\rho}^{2}(\Gamma ; V)} & \leq\|v-w\|_{L_{\rho}^{2}(\Gamma ; V)}+\left\|\mathcal{I}_{p}(w-v)\right\|_{L_{\rho}^{2}(\Gamma ; V)} \\
& \leq C_{2}\|v-w\|_{C_{\sigma}^{0}(\Gamma ; V)} .
\end{aligned}
$$

Since $w$ is arbitrary on the right-hand side, the result follows.

Copyright (c) by SIAM. Unauthorized reproduction of this article is prohibited. 
Lemma 4.3 relates the approximation error $\left(v-\mathcal{I}_{p} v\right)$ in the $L_{\rho}^{2}$-norm with the best approximation error in the weighted $C_{\sigma}^{0}$-norm for any weight $\sigma(y) \geq C_{m} e^{-(\delta y)^{2} / 4}$. We now analyze the best approximation error for a function $v: \Gamma \rightarrow V$ which admits an analytic extension in the complex plane in the region $\Sigma(\Gamma ; \tau)=\{z \in \mathbb{C}, \operatorname{dist}(z, \Gamma)<$ $\tau$ \} for some $\tau>0$. We will still denote the extension by $v$; in this case, $\tau$ represents the distance between $\Gamma \subset \mathbb{R}$ and the nearest singularity of $v(z)$ in the complex plane.

We study separately the two cases of $\Gamma$ bounded and unbounded. We start with the bounded case, in which the extra weight $\sigma$ is set equal to 1 . The following result is an immediate extension of the result given in [21, Chapter 7, section 8]

LEMMA 4.4. Given a function $v \in C^{0}(\Gamma ; V)$ which admits an analytic extension in the region of the complex plane $\Sigma(\Gamma ; \tau)=\{z \in \mathbb{C}$, $\operatorname{dist}(z, \Gamma) \leq \tau\}$ for some $\tau>0$, it holds that

$$
\min _{w \in \mathcal{P}_{p} \otimes V}\|v-w\|_{C^{0}(\Gamma ; V)} \leq \frac{2}{\varrho-1} e^{-p \log (\varrho)} \max _{z \in \Sigma(\Gamma ; \tau)}\|v(z)\|_{V},
$$

where

$$
1<\varrho=\frac{2 \tau}{|\Gamma|}+\sqrt{1+\frac{4 \tau^{2}}{|\Gamma|^{2}}}
$$

Proof. We sketch the proof for completeness. We first make a change of variables, $y(t)=y_{0}+\frac{|\Gamma|}{2} t$, where $y_{0}$ is the midpoint of $\Gamma$. Hence, $y([-1,1])=\Gamma$. We set $\tilde{v}(t)=v(y(t))$. Clearly, $\tilde{v}$ can be extended analytically in the region of the complex plane $\Sigma([-1,1] ; 2 \tau /|\Gamma|) \equiv\{z \in \mathbb{C}, \operatorname{dist}(z,[-1,1]) \leq 2 \tau /|\Gamma|\}$.

We then introduce the Chebyshev polynomials $C_{k}(t)$ on $[-1,1]$ and the expansion of $\tilde{v}:[-1,1] \rightarrow V$ as

$$
\tilde{v}(t)=\frac{a_{0}}{2}+\sum_{k=1}^{\infty} a_{k} C_{k}(t)
$$

where the Fourier coefficients $a_{k} \in V, k=0,1, \ldots$, are defined as

$$
a_{k}=\frac{1}{\pi} \int_{-\pi}^{\pi} \tilde{v}(\cos (t)) \cos (k t) d t .
$$

It is well known (see, e.g., $[21,12])$ that the series (4.2) converges in any elliptic disc $D_{\varrho} \subset \mathbb{C}$, with $\varrho>1$, delimited by the ellipse

$$
E_{\varrho}=\left\{z=t+i s \in \mathbb{C}, \quad t=\frac{\varrho+\varrho^{-1}}{2} \cos \phi, \quad s=\frac{\varrho-\varrho^{-1}}{2} \sin (\phi), \quad \phi \in[0,2 \pi)\right\}
$$

in which the function $\tilde{v}$ is analytic. Moreover (see [21] for details), we have

$$
\left\|a_{k}\right\|_{V} \leq 2 \varrho^{-k} \max _{z \in D_{\varrho}}\|\tilde{v}(z)\|_{V} .
$$

If we denote by $\Pi_{p} v \in \mathcal{P}_{p}(\Gamma) \otimes V$ the truncated Chebyshev expansion up to the polynomial degree $p$ and observe that $\left|C_{k}(t)\right| \leq 1$ for all $t \in[-1,1]$, we have

$$
\begin{aligned}
\min _{w \in \mathcal{P}_{p} \otimes V}\|v-w\|_{C^{0}(\Gamma ; V)} & \leq\left\|\tilde{v}-\Pi_{p} \tilde{v}\right\|_{C^{0}([-1,1] ; V)} \\
& \leq \sum_{k=p+1}^{\infty}\left\|a_{k}\right\|_{V} \leq \frac{2}{\varrho-1} \varrho^{-p} \max _{z \in D_{\varrho}}\|\tilde{v}(z)\|_{V} .
\end{aligned}
$$

Copyright $@$ by SIAM. Unauthorized reproduction of this article is prohibited. 
Finally, we have to link $\varrho$ to the size of the analyticity region of $\tilde{v}$. It is easy to verify that the ellipse given by

$$
\varrho=\frac{2 \tau}{|\Gamma|}\left(1+\sqrt{1+\frac{|\Gamma|^{2}}{4 \tau^{2}}}\right)
$$

is the largest ellipse that can be drawn inside $\Sigma([-1,1] ; 2 \tau /|\Gamma|)$, and this proves the stated result.

For the case of unbounded $\Gamma$ we first recall a result given in [34], and then we state in Lemma 4.6 a result tuned to our situation.

We denote by $H_{n}(y) \in P_{n}(\mathbb{R})$ the normalized Hermite polynomials

$$
H_{n}(y)=\frac{1}{\sqrt{\pi^{\frac{1}{2}} 2^{n} n !}}(-1)^{n} e^{y^{2}} \frac{\partial^{n}}{\partial y^{n}}\left(e^{-y^{2}}\right)
$$

and by $h_{n}(y)=e^{-y^{2} / 2} H_{n}(y)$ the Hermite functions. We recall that the Hermite polynomials form a complete orthonormal basis of the $L^{2}(\mathbb{R})$ space with respect to the weight $e^{-y^{2}}$, i.e.,

$$
\int_{\mathbb{R}} H_{k}(y) H_{l}(y) e^{-y^{2}} d y=\delta_{k l} .
$$

Lemma 4.5 (Hille [34]). Let $f(z)$ be an analytic function in the strip of the complex plane $\Sigma(\mathbb{R} ; \tau) \equiv\{z=(y+i w) \in \mathbb{C},-\tau \leq w \leq \tau\}$. A necessary and sufficient condition in order that the Fourier-Hermite series

$$
\sum_{k=0}^{\infty} f_{k} h_{k}(z), \quad f_{k}=\int_{\mathbb{R}} f(y) h_{k}(y) d y,
$$

shall exist and converge to the sum $f(z)$ in $\Sigma(\mathbb{R} ; \tau)$ is that for every $\beta, 0 \leq \beta<\tau$, there exists a finite positive $C(\beta)$ such that

$$
|f(y+i w)| \leq C(\beta) e^{-|y| \sqrt{\beta^{2}-w^{2}}}, \quad-\infty<y<\infty, \quad-\beta \leq w \leq \beta .
$$

Moreover, the following bound for the Fourier coefficients holds:

$$
\left|f_{n}\right| \leq C e^{-\tau \sqrt{2 n+1}}
$$

In particular, the previous result tells us that, in order to have exponential decay of the Fourier coefficients $f_{n}$, we not only need $f(z)$ to be analytic in $\Sigma(\mathbb{R} ; \tau)$ but also must require that it decay on the real line, for $y \rightarrow \infty$, at least as $e^{-\tau|y|}$.

We now introduce two weights: the exponential $\sigma=e^{-\alpha|y|}$, for some $\alpha>0$, and the Gaussian $G=e^{-(\delta y)^{2} / 4}$. We recall that Lemma 4.3 holds for both. We will assume that the function $v$ is in the space $C_{\sigma}^{0}(\Gamma ; V)$, but we will measure the best approximation error in the weaker norm $C_{G}^{0}(\Gamma ; V)$, with Gaussian weight, so that we can use the result from Hille given in Lemma 4.5. The following lemma holds.

Lemma 4.6. Let $v$ be a function in $C_{\sigma}^{0}(\mathbb{R} ; V)$. We suppose that $v$ admits an analytic extension in the strip of the complex plane $\Sigma(\mathbb{R} ; \tau)=\{z \in \mathbb{C}, \operatorname{dist}(z, \mathbb{R}) \leq \tau\}$ for some $\tau>0$, and that

$$
\forall z=(y+i w) \in \Sigma(\mathbb{R} ; \tau), \quad \sigma(y)\|v(z)\|_{V} \leq C_{v}(\tau) .
$$

Copyright $@$ by SIAM. Unauthorized reproduction of this article is prohibited. 
Then, for any $\delta>0$, there exist a constant $C$, independent of $p$, and a function $\Theta(p)=O(\sqrt{p})$ such that

$$
\min _{w \in \mathcal{P}_{p} \otimes V} \max _{y \in \mathbb{R}}\left|\|v(y)-w(y)\|_{V} e^{-\frac{(\delta y)^{2}}{4}}\right| \leq C \Theta(p) e^{-\tau \delta \sqrt{p}} .
$$

Proof. We introduce the change of variable $t=\delta y / \sqrt{2}$ and denote $\tilde{v}(t)=v(y(t))$. Observe that $\tilde{v} \in C_{\tilde{\sigma}}^{0}(\mathbb{R} ; V)$ with weight $\tilde{\sigma}=e^{-\sqrt{2} \frac{\alpha}{\delta}|t|}$. We consider the expansion of $\tilde{v}$ in Hermite polynomials

$$
\tilde{v}(t)=\sum_{k=0}^{\infty} v_{k} H_{k}(t), \quad \text { where } v_{k} \in V, \quad v_{k}=\int_{\mathbb{R}} \tilde{v}(t) H_{k}(t) e^{-t^{2}} d t .
$$

We now set $f(z)=\tilde{v}(z) e^{-\frac{z^{2}}{2}}$. Observe that the Hermite expansion of $f$ as defined in (4.4) has the same Fourier coefficients as the expansion of $\tilde{v}$ defined in (4.7). Indeed

$$
f_{k}=\int_{\mathbb{R}} f(t) h_{k}(t) d t=\int_{\mathbb{R}} \tilde{v}(t) H_{k}(t) e^{-t^{2}} d t=v_{k} .
$$

Clearly, $f(z)$ is analytic in the strip $\Sigma\left(\mathbb{R} ; \frac{\tau \delta}{\sqrt{2}}\right)$, being the product of analytic functions. Moreover,

$$
\|f(y+i w)\|_{V}=\left|e^{-\frac{(y+i w)^{2}}{2}}\right|\|\tilde{v}(z)\|_{V} \leq e^{-\frac{y^{2}-w^{2}}{2}} e^{\sqrt{2} \frac{\alpha}{\delta}|y|} C_{v}(\tau) .
$$

Setting

$$
C(\beta)=\max _{\substack{-\infty<y<\infty \\-\beta \leq w \leq \beta}} \exp \left\{-\frac{y^{2}-w^{2}}{2}+\sqrt{2} \frac{\alpha}{\delta}|y|+|y| \sqrt{\beta^{2}-w^{2}}\right\},
$$

which is bounded for all $-\frac{\tau \delta}{\sqrt{2}} \leq \beta \leq \frac{\tau \delta}{\sqrt{2}}$, the function $f(z)$ satisfies the hypotheses of Lemma 4.5. Hence the Hermite series converges in $\Sigma\left(\mathbb{R} ; \frac{\tau \delta}{\sqrt{2}}\right)$ and the Fourier coefficients $v_{k}$ behave as in (4.6). We choose $w \in \mathcal{P}_{p} \otimes V$ as the truncated Hermite expansion of $v$, up to degree $p: \tilde{w}(t)=\Pi_{p} \tilde{v}(t)=\sum_{k=0}^{p} v_{k} H_{k}(t)$. We have

$$
\begin{aligned}
E_{p}(v) & =\min _{w \in \mathcal{P}_{p} \otimes V} \max _{y \in \mathbb{R}}\left|\|v(y)-w(y)\|_{V} e^{-\frac{(\delta y)^{2}}{4}}\right| \\
& \leq \max _{t \in \mathbb{R}}\left|\left\|\tilde{v}(t)-\Pi_{p} \tilde{v}(t)\right\|_{V} e^{-\frac{t^{2}}{2}}\right| \leq \max _{t \in \mathbb{R}}\left\|\sum_{k=p+1}^{\infty} v_{k} h_{k}(t)\right\|_{V} .
\end{aligned}
$$

It is well known (see, e.g., [11]) that the Hermite functions $h_{k}(t)$ satisfy $\left|h_{k}(t)\right|<1$ for all $t \in \mathbb{R}$ and all $k=0,1, \ldots$. Hence, the previous series can be bound as

$$
E_{p}(v) \leq \sum_{k=p+1}^{\infty}\left\|v_{k}\right\|_{V} \leq C \sum_{k=p+1}^{\infty} e^{-\frac{\tau \delta}{\sqrt{2}} \sqrt{2 k+1}} .
$$

We now use the following bound for the series above, whose proof can be found in [7, Appendix, Lemma A.2]:

$$
\forall r \in \mathbb{R}^{+}, r<1, \quad \sum_{k=p+1}^{\infty} r^{\sqrt{2 k+1}} \leq a^{\sqrt{p+1}} \frac{(2 \sqrt{p+1}+1)(1-a)+2}{a(1-a)^{2}}, \quad a=r^{\sqrt{2}},
$$

and this concludes the proof. 
We are now ready to prove Theorem 4.1.

Proof of Theorem 4.1. The error naturally splits into $\epsilon=\left(u-u_{h}\right)+\left(u_{h}-u_{h, p}\right)$. The first term depends on the space discretization only and can be estimated easily; indeed, the function $u_{h}$ is the orthogonal projection of $u$ onto the subspace $L_{\rho}^{2}(\Gamma) \otimes H_{0}^{1}(D)$ with respect to the inner product $\int_{\Gamma \times D} \rho a|\nabla \cdot|^{2}$. Hence

$$
\begin{aligned}
\left\|u-u_{h}\right\|_{L_{\rho}^{2}(\Gamma) \otimes H_{0}^{1}(D)} & \leq \frac{1}{\sqrt{a_{\min }}}\left(\int_{\Gamma \times D} \rho a\left|\nabla\left(u-u_{h}\right)\right|^{2}\right)^{\frac{1}{2}} \\
& \leq \frac{1}{\sqrt{a_{\min }}} \inf _{v \in L_{\rho}^{2}(\Gamma) \otimes H_{h}(D)}\left(\int_{\Gamma \times D} \rho a|\nabla(u-v)|^{2}\right)^{\frac{1}{2}} .
\end{aligned}
$$

The second term $u_{h}-u_{h, p}$ is an interpolation error. We recall, indeed, that $u_{h, p}=$ $\mathcal{I}_{p} u_{h}$. To lighten the notation, we will drop the subscript $h$, it being understood that we work on the semidiscrete solution. We recall, moreover, that $u_{h}$ has the same regularity as the exact solution $u$ with respect to $y$.

To analyze this term we employ a one-dimensional argument. We first pass from the norm $L_{\rho}^{2}$ to $L_{\hat{\rho}}^{2}$ :

$$
\left\|u-\mathcal{I}_{p} u\right\|_{L_{\rho}^{2} \otimes H_{0}^{1}} \leq\left\|\frac{\rho}{\hat{\rho}}\right\|_{L^{\infty}(\Gamma)}^{\frac{1}{2}}\left\|u-\mathcal{I}_{p} u\right\|_{L_{\hat{\rho}}^{2} \otimes H_{0}^{1}} .
$$

Here we adopt the same notation as in section 3 ; namely, we indicate with $\bullet_{n}$ a quantity relative to the direction $y_{n}$ and with $\bullet_{n}^{*}$ the analogous quantity relative to all other directions $y_{j}, j \neq n$. We focus on the first direction $y_{1}$ and define an interpolation operator $\mathcal{I}_{1}: C_{\sigma_{1}}^{0}\left(\Gamma_{1} ; L_{\hat{\rho}_{1}^{*}}^{2} \otimes H_{0}^{1}\right) \rightarrow L_{\hat{\rho}_{1}}^{2}\left(\Gamma_{1} ; L_{\hat{\rho}_{1}^{*}}^{2} \otimes H_{0}^{1}\right)$,

$$
\mathcal{I}_{p_{1}} v\left(y_{1}, y_{1}^{*}, x\right)=\sum_{k=1}^{p_{1}+1} v\left(y_{1, k}, y_{1}^{*}, x\right) l_{1, k}\left(y_{1}\right)
$$

Then the global interpolant $\mathcal{I}_{p}$ can be written as the composition of two interpolation operators $\mathcal{I}_{p}=\mathcal{I}_{1} \circ \mathcal{I}_{p}^{(1)}$, where $\mathcal{I}_{p}^{(1)}$ is the interpolation operator in all directions $y_{2}, y_{3}, \ldots, y_{N}$ except $y_{1}: \mathcal{I}_{p}^{(1)}: C_{\sigma_{1}^{*}}^{0}\left(\Gamma_{1}^{*} ; H_{0}^{1}\right) \rightarrow L_{\hat{\rho}_{1}^{*}}^{2}\left(\Gamma_{1}^{*} ; H_{0}^{1}\right)$. We then have

$$
\left\|u-\mathcal{I}_{p} u\right\|_{L_{\hat{\rho}}^{2} \times H_{0}^{1}} \leq \underbrace{\left\|u-\mathcal{I}_{1} u\right\|_{L_{\hat{\rho}}^{2} \times H_{0}^{1}}}_{\mathrm{I}}+\underbrace{\left\|\mathcal{I}_{1}\left(u-\mathcal{I}_{p}^{(1)} u\right)\right\|_{L_{\hat{\rho}}^{2} \times H_{0}^{1}}}_{\mathrm{II}} .
$$

Let us bound the first term. We think of $u$ as a function of $y_{1}$ with values in a Hilbert space $V, u \in L_{\hat{\rho}_{1}}^{2}\left(\Gamma_{1} ; V\right)$, where $V=L_{\hat{\rho}_{1}^{*}}^{2}\left(\Gamma_{1}^{*}\right) \otimes H_{0}^{1}(D)$. Under Assumption 2 in section 3 and the choice of $\hat{\rho}$ given in (3.3), the following inclusions hold true:

$$
C_{\sigma_{1}}^{0}\left(\Gamma_{1} ; V\right) \subset C_{G_{1}}^{0}\left(\Gamma_{1} ; V\right) \subset L_{\hat{\rho}_{1}}^{2}\left(\Gamma_{1} ; V\right)
$$

with $\sigma_{1}=G_{1}=1$ if $\Gamma_{1}$ is bounded and $\sigma_{1}=e^{-\alpha_{1}\left|y_{1}\right|}, G_{1}=e^{-\frac{\left(\delta_{1} y_{1}\right)^{2}}{4}}$ if $\Gamma_{1}$ is unbounded. We know also from Lemma 4.2 that the interpolation operator $\mathcal{I}_{1}$ is continuous both as an operator from $C_{\sigma_{1}}^{0}\left(\Gamma_{1} ; V\right)$ with values in $L_{\hat{\rho}_{1}}^{2}\left(\Gamma_{1} ; V\right)$ and from $C_{G_{1}}^{0}\left(\Gamma_{1} ; V\right)$ in $L_{\hat{\rho}_{1}}^{2}\left(\Gamma_{1} ; V\right)$. In particular, we can estimate

$$
\mathrm{I}=\left\|u-\mathcal{I}_{1} u\right\|_{L_{\hat{\rho}_{1}}^{2}\left(\Gamma_{1} ; V\right)} \leq C_{2} \inf _{w \in \mathcal{P}_{p_{1}} \otimes V}\|u-w\|_{C_{G_{1}}^{0}(\Gamma ; V)} .
$$


To bound the best approximation error in $C_{G_{1}}^{0}(\Gamma ; V)$, in the case of $\Gamma_{1}$ bounded we use Lemma 4.4, whereas if $\Gamma_{1}$ is unbounded, we employ Lemma 4.6 and the fact that $u \in C_{\sigma_{1}}^{0}\left(\Gamma_{1} ; V\right)$ (see Lemma 3.1). In both cases, we need the analyticity result, for the solution $u$, stated in Lemma 3.2. Putting everything together, we can say that

$$
\mathrm{I} \leq \begin{cases}C e^{-r_{1} p_{1}}, & \Gamma_{1} \text { bounded } \\ C \Theta\left(p_{1}\right) e^{-r_{1} \sqrt{p_{1}}}, & \Gamma_{1} \text { unbounded }\end{cases}
$$

the value of $r_{1}$ being specified in Lemmas 4.4 and 4.6. To bound the term II, we use Lemma 4.2:

$$
\mathrm{II} \leq C_{1}\left\|u-\mathcal{I}_{p}^{(1)} u\right\|_{C_{\sigma_{1}}^{0}\left(\Gamma_{1} ; V\right)} .
$$

The term on the right-hand side is again an interpolation error. Thus we have to bound the interpolation error in all the other $N-1$ directions, uniformly with respect to $y_{1}$ (in the weighted norm $C_{\sigma_{1}}^{0}$ ). We can proceed iteratively, defining an interpolation $\mathcal{I}_{2}$, bounding the resulting error in the direction $y_{2}$, and so on.

4.I. Convergence of Moments. In some cases one might be interested only in computing the first few moments of the solution, namely, $E\left[u^{m}\right], m=1,2, \ldots$ We show in the next two lemmas that the error in the first two moments, measured in a suitable spatial norm, is bounded by the mean square error $\left\|u-u_{h, p}\right\|_{L_{\rho}^{2} \otimes H_{0}^{1}}$, which, due to Theorem 4.1, is exponentially convergent with respect to the polynomial degree $p$ employed in the $y$ directions. In particular, without extra regularity assumptions on the solution $u$ of the problem, we have optimal convergence for the error in the mean value (first moment) measured in $L^{2}(D)$ or $H^{1}(D)$ and for the error in the second moment measured in $L^{1}(D)$.

LEMMA 4.7 (approximation of mean value).

$$
\left\|E\left[u-u_{h, p}\right]\right\|_{V(D)} \leq\left\|u-u_{h, p}\right\|_{L_{\rho}^{2}(\Gamma) \otimes V(D)} \text {, with } V(D)=L^{2}(D) \text { or } H^{1}(D) .
$$

The proof is immediate and omitted. Although the previous estimate implies exponential convergence with respect to $p$, under the assumptions of Theorem 4.1, the above estimate is suboptimal and can be improved by a duality argument (see [4] and Remark 5.2 in [5]).

LEMMA 4.8 (approximation of the second moment).

$$
\left\|E\left[u^{2}-u_{h, p}^{2}\right]\right\|_{L^{1}(D)} \leq C\left\|u-u_{h, p}\right\|_{L_{\rho}^{2}(\Gamma) \otimes L^{2}(D)},
$$

with $C$ independent of the discretization parameters $h$ and $p$.

Proof. We have

$$
\begin{aligned}
\left\|E\left[u^{2}-u_{h, p}^{2}\right]\right\|_{L^{1}(D)} & =\left\|E\left[\left(u-u_{h, p}\right)\left(u+u_{h, p}\right)\right]\right\|_{L^{1}(D)} \\
& \leq\left\|u-u_{h, p}\right\|_{L_{\rho}^{2}(\Gamma) \otimes L^{2}(D)}\left\|u+u_{h, p}\right\|_{L_{\rho}^{2}(\Gamma) \otimes L^{2}(D)} \\
& \leq\left\|u-u_{h, p}\right\|_{L_{\rho}^{2}(\Gamma) \otimes L^{2}(D)}\left(\|u\|_{L_{\rho}^{2}(\Gamma) \otimes L^{2}(D)}+\left\|u_{h, p}\right\|_{L_{\rho}^{2}(\Gamma) \otimes L^{2}(D)}\right) .
\end{aligned}
$$

The term $\left\|u_{h, p}\right\|_{L_{\rho}^{2} \otimes L^{2}}$ can be bounded as

$$
\left\|u_{h, p}\right\|_{L_{\rho}^{2}(\Gamma) \otimes L^{2}(D)}=\left\|\mathcal{I}_{p} u_{h}\right\|_{L_{\rho}^{2}(\Gamma) \otimes L^{2}(D)} \leq C_{1}\left\|u_{h}\right\|_{C_{\sigma}^{0}\left(\Gamma ; L^{2}(D)\right)} \leq C\left(f, a_{\min }\right),
$$

where we have used the boundedness of the interpolation operator $\mathcal{I}_{p}$ stated in Lemma 4.2. The last inequality follows from the fact that the semidiscrete solution $u_{h}$ is the 
orthogonal projection of the exact solution $u$ onto the subspace $H_{h}$ with respect to the energy inner product; hence

$$
\left\|\sqrt{a(y)} \nabla u_{h}(y)\right\|_{L^{2}(D)} \leq\|\sqrt{a(y)} \nabla u(y)\|_{L^{2}(D)} \quad \forall y \in \Gamma,
$$

and the last term can be controlled in terms of $a_{\min }$ and the forcing term, $f$.

Similarly, it is possible to estimate the approximation error in the covariance function of the solution $u$.

On the other hand, to estimate the convergence rate of the error in higher order moments, or of the second moment in higher norms, we need extra regularity assumptions on the solution to ensure proper integrability and then be able to use analyticity.

In [44] it is also shown that controlling the mean square error $\left\|u-u_{h, p}\right\|_{L_{\rho}^{2} \otimes H_{0}^{1}}$ is enough to have a control on all statistical moments of a locally Lipschitz functional of the solution. More precisely, let $\zeta: H_{0}^{1}(D) \rightarrow \mathbb{R}$ be a possibly nonlinear functional, such that $\zeta(0)=0$. Given $R>0$, we assume that $\zeta$ is Lipschitz in the ball $\mathcal{B}\left(H_{0}^{1}(D), R\right)$ of radius $R$; i.e., there exists a constant $\Xi_{R}$ such that

$$
|\zeta(u)-\zeta(v)| \leq \Xi_{R}\|u-v\|_{H_{0}^{1}} \quad \forall u, v \in \mathcal{B}\left(H_{0}^{1}(D), R\right) .
$$

Provided that both the exact solution $u$ and the discrete one $u_{h, p}$ are bounded uniformly in $\Gamma$, i.e., $\|u(y)\|_{H_{0}^{1}} \leq \Lambda$ and $\left\|u_{h, p}(y)\right\|_{H_{0}^{1}} \leq \Lambda$ for all $y \in \Gamma$, then it can be shown [44] that

$$
E\left[\zeta(u)^{q}-\zeta\left(u_{h, p}\right)^{q}\right] \leq q \Xi_{\Lambda}^{q} \Lambda^{q-1}\left\|u-u_{h, p}\right\|_{L_{\rho}^{2} \otimes H_{0}^{1}} \quad \text { for any } 1 \leq q<\infty .
$$

Hence the error on the $q$ th moment of the random variable $\zeta(u)$ will be exponentially convergent, however with a constant that depends on $q$.

5. Numerical Examples. This section illustrates the convergence of the collocation method for a stochastic elliptic problem in two dimensions. The computational results are in accordance with the convergence rate predicted by the theory.

The problem to solve is

$$
\begin{aligned}
& -\nabla \cdot(a \nabla u)=0 \quad \text { on } \Omega \times D, \\
& u=0 \quad \text { on } \Omega \times \partial D_{D}, \\
& -a \partial_{n} u=1 \quad \text { on } \Omega \times \partial D_{N} \text {, } \\
& \partial_{n} u=0 \quad \text { on } \Omega \times\left(\partial D-\left(\partial D_{D} \cup \partial D_{N}\right)\right),
\end{aligned}
$$

with

$$
\begin{aligned}
& D=\left\{(x, z) \in \mathbb{R}^{2}:-1.5 \leq x \leq 0,-0.4 \leq z \leq 0.8\right\} \\
& \partial D_{D}=\left\{(x, z) \in \mathbb{R}^{2}:-1 \leq x \leq-0.5, z=0.8\right\} \\
& \partial D_{N}=\left\{(x, z) \in \mathbb{R}^{2}:-1.5 \leq x \leq 0, z=-0.4\right\}
\end{aligned}
$$

cf. Figure 1.

The random diffusivity coefficient is a nonlinear function of the random vector $Y$, namely,

$$
\begin{aligned}
a(\omega, x)=a_{\min }+\exp \{ & {\left[Y_{1}(\omega) \cos (\pi z)+Y_{3}(\omega) \sin (\pi z)\right] e^{-\frac{1}{8}} } \\
+ & {\left.\left[Y_{2}(\omega) \cos (\pi x)+Y_{4}(\omega) \sin (\pi x)\right] e^{-\frac{1}{8}}\right\} . }
\end{aligned}
$$

Copyright $@$ by SIAM. Unauthorized reproduction of this article is prohibited. 


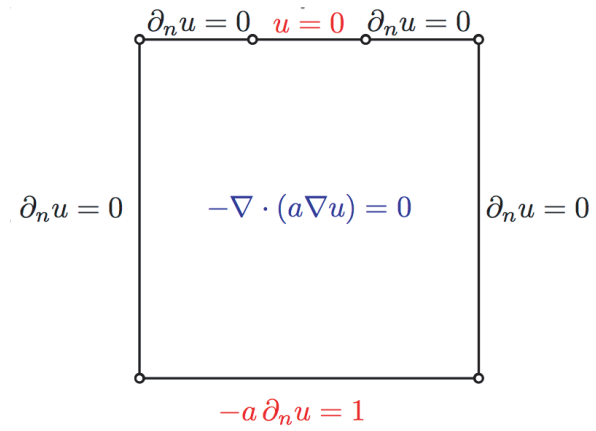

Fig. I Geometry and boundary conditions for the numerical example.

Here $a_{\text {min }}=1 / 100$, and the real random variables $Y_{n}, n=1, \ldots, 4$, are independent and identically distributed with mean value zero and unit variance. To illustrate the behavior of the collocation method with either unbounded or bounded random variables $Y_{n}$, this section presents two different cases, corresponding to either Gaussian or uniform densities. The corresponding collocation points are then Cartesian products determined by the roots of either Hermite or Legendre polynomials.

Observe that the collocation method requires only the solution of uncoupled deterministic problems in the collocation points, even in the presence of a diffusivity coefficient which depends nonlinearly on the random variables as in (5.1). This is a great advantage with respect to the classical stochastic Galerkin finite element method as considered in [4] or [43] (see also the considerations given in section 2.1). Observe, moreover, how easily the collocation method can deal with unbounded random variables.

Figure 2 shows some realizations of the logarithm of the diffusivity coefficient, while Figures 3 and 4 show the mean and variance of the corresponding solutions. The finite element space for spatial discretization is the span of continuous functions that are piecewise polynomials with degree five over a triangulation with 1178 triangles and 642 vertices; see Figure 5. This triangulation has been adaptively graded to control the singularities at the boundary points $(-1,0.8)$ and $(-0.5,0.8)$. These singularities occur where the Dirichlet and Neumann boundaries meet, and they essentially behave like $\sqrt{r}$, with $r$ being the distance to the closest singularity point.

To study the convergence of the tensor product collocation method, we increase the order $p$ for the approximating polynomial spaces, $\mathcal{P}_{p}(\Gamma)$, following the adaptive algorithm described on page 1287 of [5]. This adaptive algorithm increases the tensor polynomial degree with an anisotropic strategy: it increases the order of approximation in one direction as much as possible before considering the next direction.

The computational results for the $H_{0}^{1}(D)$ approximation error in the expected value, $E[u]$, are shown on Figure 6 , while those corresponding to the approximation of the second moment, $E\left[u^{2}\right]$, are shown on Figure 7 . To estimate the computational error in the $i$ th direction, corresponding to a multi-index $p=\left(p_{1}, \ldots, p_{i}, \ldots, p_{N}\right)$, we approximate it by $E[e] \approx E\left[u_{h, p}-u_{h, \tilde{p}}\right]$, with $\tilde{p}=\left(p_{1}, \ldots, p_{i}+1, \ldots, p_{N}\right)$. We proceed similarly for the error in the approximation of the second moment.

As expected, the estimated approximation error decreases exponentially fast as the polynomial order increases, for both the computation of $E[u]$ and $E\left[u^{2}\right]$, with either Gaussian or uniform probability densities. 

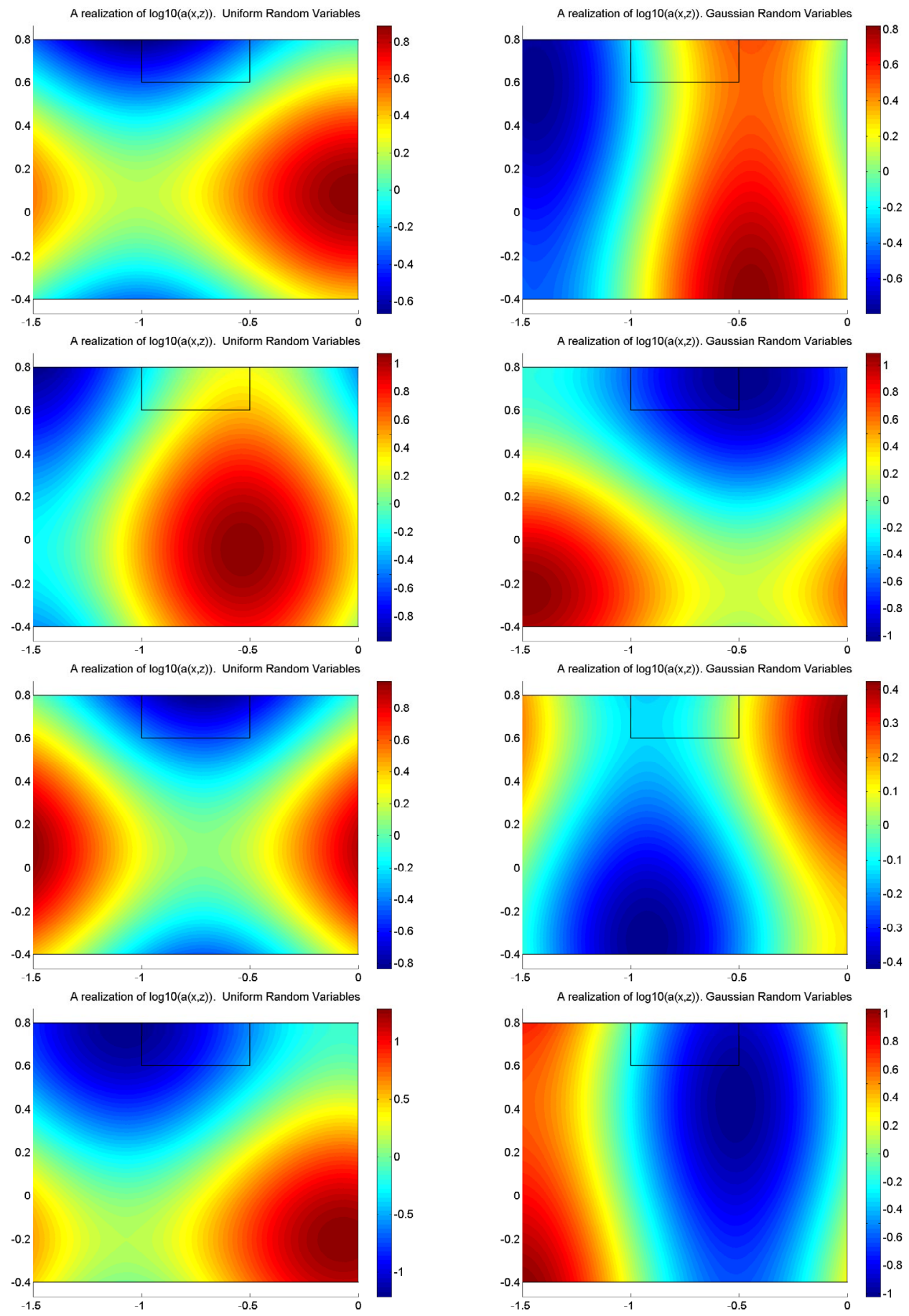

Fig. 2 Some realizations of $\log (a)$. 


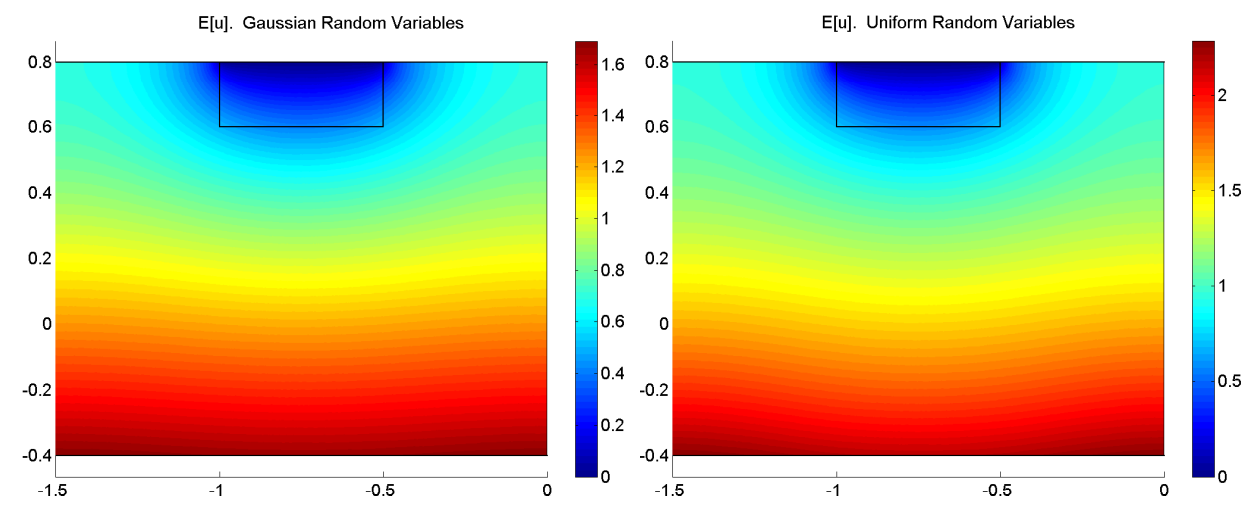

Fig. 3 Results for the computation of the expected value for the solution, $E[u]$.
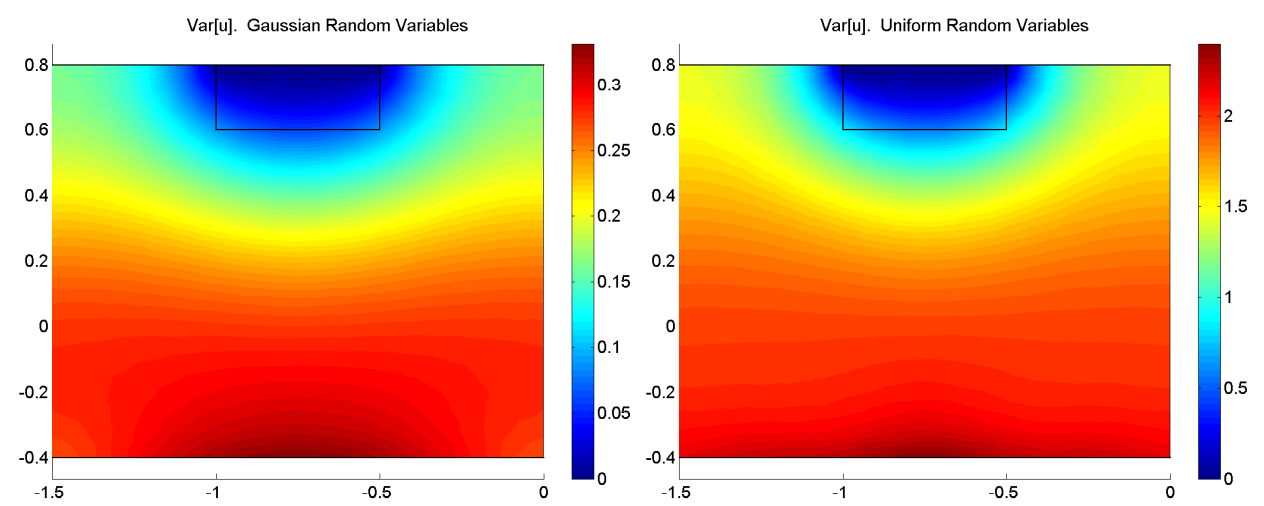

Fig. 4 Results for the computation of the variance of the solution, $\operatorname{Var}[u]$.

6. Further Developments. After the works [61, 7] were published, there have been a lot of developments in stochastic collocation methods, mainly concerning effective collocation strategies for problems that depend on a moderately large number of random variables (high dimensionality of the probability space).

The stochastic collocation method proposed in section 2 is based on a tensor product polynomial approximation and interpolates the semidiscrete solution $u_{h}$ of problem (2.1) on a full tensor grid obtained by tensorization of one-dimensional Gaussian abscissas. This approach can be computationally expensive if the number $N$ of random variables needed to describe the input data is moderately large. This drawback is often called the curse of dimensionality, which, in this context, refers to the exponential growth in the required amount of computational work with respect to the number of input random variables.

The immediate consequence of the curse of dimensionality is that the convergence rate of the error with respect to the total number of collocation points, a quantity directly related to the overall computational cost, is very poor for $N$ large. To fix ideas, let us consider the case of bounded random variables and equal polynomial degree $p$ in each direction. We assume, moreover, that the exponential convergence rate $r_{n}$ in Theorem 4.1 is practically the same $r_{n} \approx r$ for all $n=1, \ldots, N$ and 

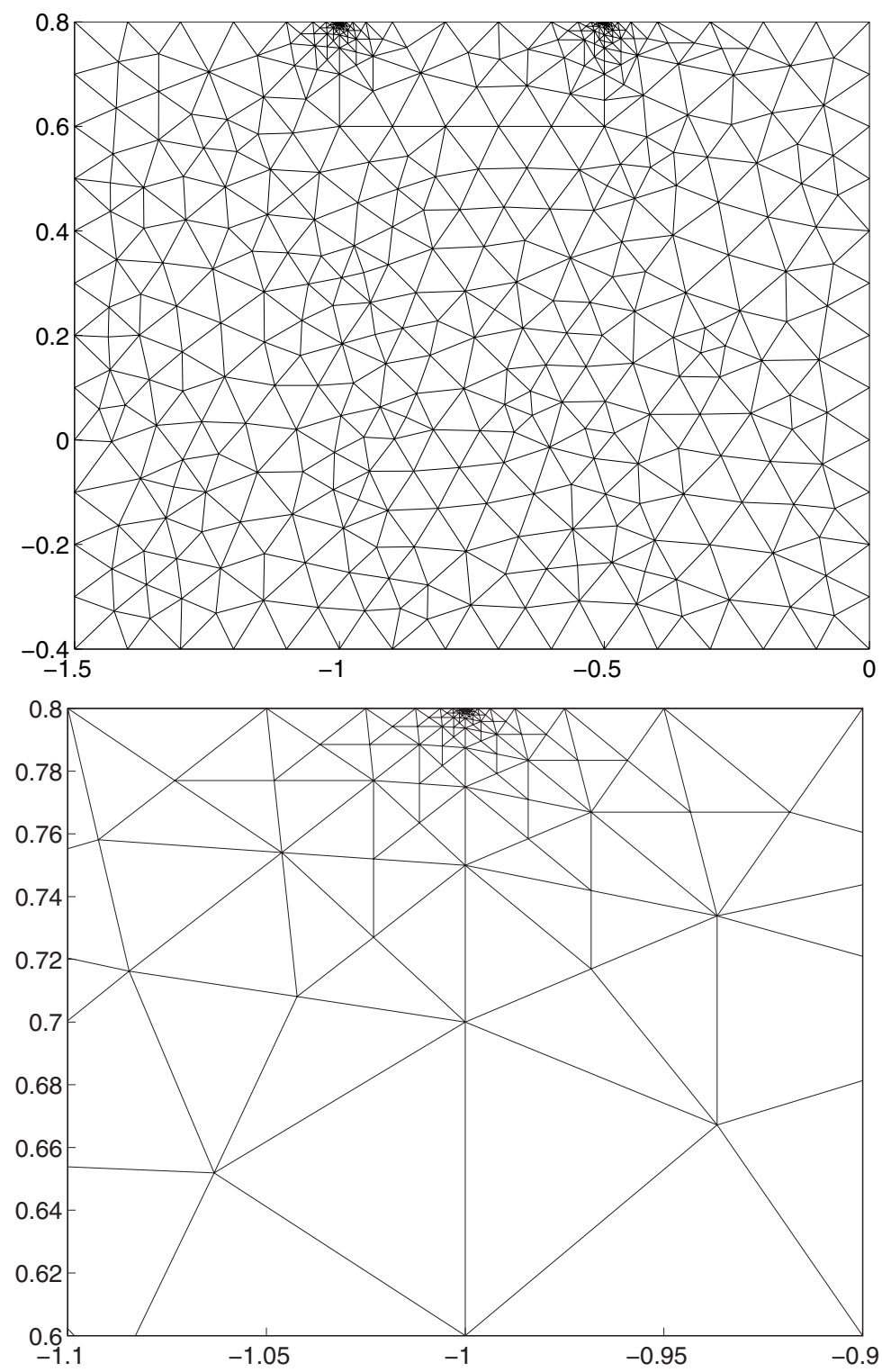

Fig. 5 Top: Unstructured grid for the spatial discretization. The corresponding finite element spaces are the span of continuous functions that are piecewise polynomials with degree five. Bottom: Detail of the mesh refinement near the left singularity.

denote by err $=\left\|u_{h}-u_{h, p}\right\|_{L_{\rho}^{2} \otimes H_{0}^{1}}$ the error between the semidiscrete solution $u_{h}$ and its stochastic collocation approximation. Then Theorem 4.1 states that the error err is exponentially decaying in $p$, i.e., $\operatorname{err} \leq C \exp \{-r p\}$. However, the number of collocation points in the full tensor grid is $\eta=(1+p)^{N}$, which leads to an error versus cost relation of $e r r \leq \tilde{C} \exp \left\{-r \eta^{1 / N}\right\}$. Observe that if $\eta \ll e^{N}$, which is likely to happen if $N$ is large, then $\eta^{1 / N} \cong 1+\log (\eta) / N$ and the effective rate is algebraic, 

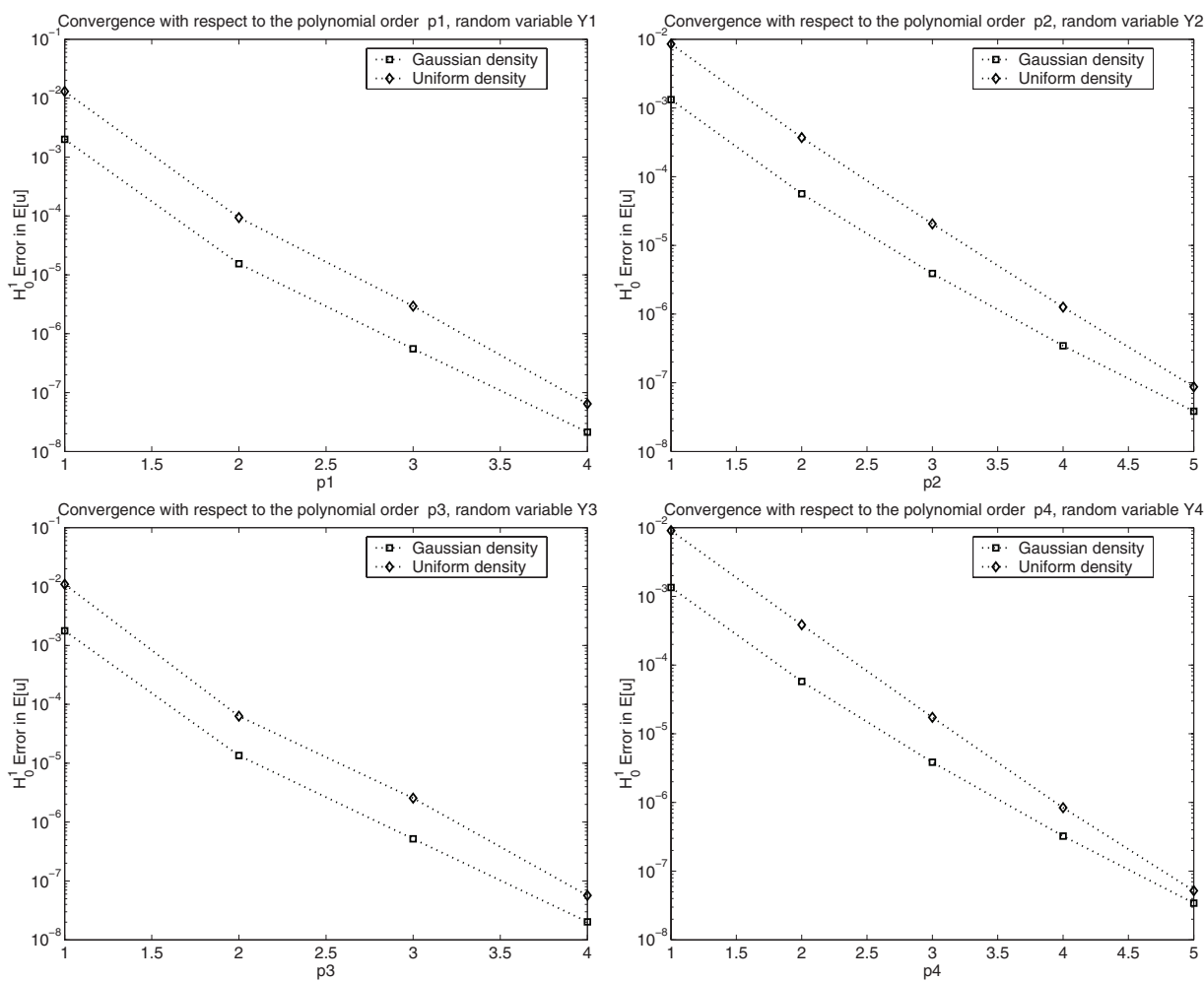

Fig. 6 Convergence results for the approximation of the expected value, $E[u]$.

rather than exponential,

$$
e r r \leq C \eta^{-r / N},
$$

and can easily become worse than the Monte Carlo convergence rate, $e r r \approx \eta^{-1 / 2}$.

On the other hand, it is well known that to build a multivariate approximation or a quadrature formula for a smooth function, a full tensor grid is often unnecessary and much smaller sparse grids can be used instead. This idea dates back to Smolyak [53] and has been exploited by several authors in different contexts in the last three decades (see, e.g., $[15,48]$ and the references therein).

In the framework of PDEs with random input data, the sparse grid stochastic collocation method has been proposed in [61] and analyzed in [46] (see also [29, 26]). It naturally requires solving uncoupled deterministic problems as in the Monte Carlo method while keeping the number of evaluation points much smaller than the full tensor stochastic collocation version introduced in section 2 . The sparse grid approach, and in particular its anisotropic version proposed in [45], greatly reduces the curse of dimensionality and may be expected to be competitive with the classical Monte Carlo method even for moderately large dimensionality. Therefore, it is of major practical relevance to understand in which situations the sparse grid stochastic collocation method is more efficient than the Monte Carlo method.

In this section we review the construction of a sparse grid approximation. It builds upon stochastic collocation on a sequence of tensor grids and in this respect 

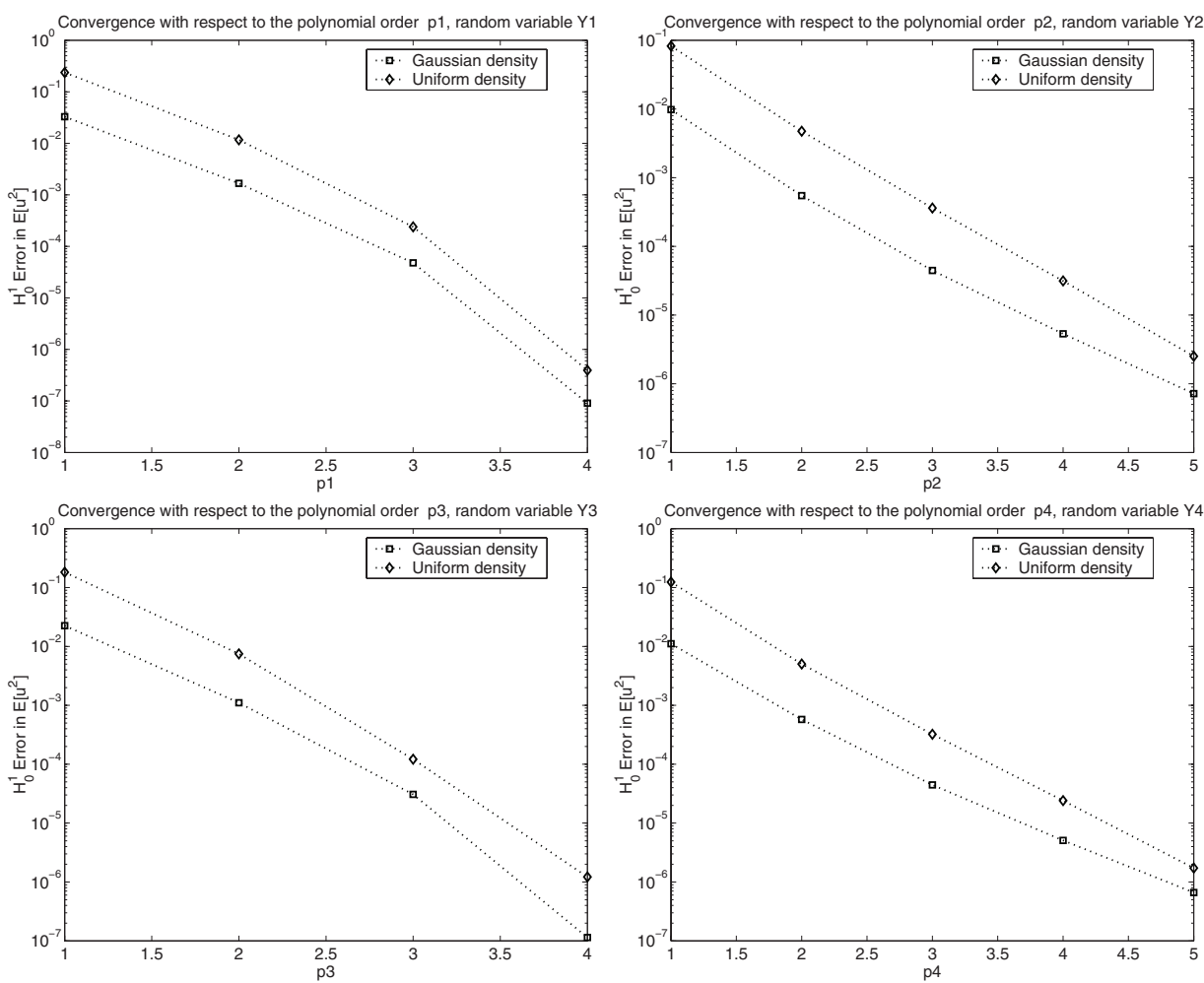

Fig. 7 Convergence results for the approximation of the second moment, $E\left[u^{2}\right]$.

is a generalization of the method presented in section 2. To provide insight into its performance, section 6.2 states error estimates derived first in $[46,45]$ for the fully discrete solution, analyzing the computational efficiency of the sparse grid stochastic collocation method in terms of the number of collocation points (deterministic problems to solve). In particular, the estimates derived in [46] demonstrate at least algebraic convergence with respect to the total number of collocation points of the type $\operatorname{err} \leq C \eta^{-r /(1+\log (N))}$, thus proving a highly reduced curse of dimensionality with respect to full tensor collocation.

Computational evidence shown in section 6.4 complements the theory and shows the effectiveness of the sparse grid stochastic collocation method compared to full tensor stochastic collocation, the Monte Carlo method, and stochastic Galerkin as defined in (2.4) (see also [32, 4, 27, 62] and the references therein).

6.I. Sparse Grid Stochastic Collocation Approximation. We present here a generalization of the classical Smolyak construction (see, e.g., [53, 9]) to build a multivariate polynomial approximation on a sparse grid. As in Lemma 4.2, for each direction $y_{n}$ we introduce a sequence of one-dimensional polynomial interpolant operators of increasing order: $\mathcal{I}_{n}^{m(i)}: C_{\sigma}^{0}\left(\Gamma_{n} ; V\right) \rightarrow \mathbb{P}_{m(i)-1}\left(\Gamma_{n}\right) \otimes V$. Here $i \geq 1$ denotes the level of approximation and $m(i)$ the number of collocation points used to build the interpolation at level $i$, with the requirement that $m(1)=1$ and $m(i)<m(i+1)$ for $i \geq 1$. In addition, let $m(0)=0$ and $\mathcal{I}_{n}^{m(0)}=0$. Here the collocation abscissas 
$\left\{y_{n, j}^{(i)}, j=1, \ldots, m(i)\right\}$ for the one-dimensional interpolation formula $\mathcal{I}_{n}^{m(i)}$ are Gauss points with respect to the weight $\hat{\rho}_{n}$, that is, the zeros of the $\hat{\rho}_{n}$-orthogonal polynomial $q_{n, m(i)}$. Further, we introduce the difference operators

$$
\Delta_{n}^{m(i)}=\mathcal{I}_{n}^{m(i)}-\mathcal{I}_{n}^{m(i-1)} .
$$

Given an integer $w \geq 0$ called the approximation level and a multi-index $\mathbf{i}=\left(i_{1}, \ldots, i_{N}\right)$ $\in \mathbb{N}_{+}^{N}, \mathbf{i} \geq \mathbf{1}$, we introduce a function $g: \mathbb{N}_{+}^{N} \rightarrow \mathbb{N}$ strictly increasing in each argument and define a sparse grid approximation of the semidiscrete solution $u_{h}$ of problem (2.1) as

$$
\mathcal{S}_{w}^{m, g}\left[u_{h}\right]=\sum_{\mathbf{i} \in \mathbb{N}_{+}^{N}: g(\mathbf{i}) \leq w} \bigotimes_{n=1}^{N} \Delta_{n}^{m\left(i_{n}\right)}\left(u_{h}\right)
$$

Formula (6.1) can be equivalently written as

$$
\mathcal{S}_{w}^{m, g}\left[u_{h}\right]=\sum_{\mathbf{i} \in \mathbb{N}_{+}^{N}: g(\mathbf{i}) \leq w} c(\mathbf{i}) \bigotimes_{n=1}^{N} \mathcal{I}_{n}^{m\left(i_{n}\right)}\left(u_{h}\right), \quad \text { with } c(\mathbf{i})=\sum_{\substack{\mathbf{j} \in\{0,1\}^{N} \\ g(\mathbf{i}+\mathbf{j}) \leq w}}(-1)^{|\mathbf{j}|}
$$

From the previous expression, we see that the sparse grid approximation is obtained as a linear combination of full tensor product interpolations, as defined in section 2 . However, the constraint $g(\mathbf{i}) \leq w$ in $(6.2)$ is typically chosen so as to forbid the use of tensor grids of high degree in all directions at the same time.

Observe moreover that whenever $\mathbf{i}$ is such that $\left\{\mathbf{j} \in\{0,1\}^{N}: g(\mathbf{i}+\mathbf{j}) \leq w\right\}=$ $\{0,1\}^{N}$, then $c(\mathbf{i})=0$, so there may be many terms in the sum in (6.2) that do not need to be computed. Formula (6.2) implies evaluation of the function $u_{h}$ in a finite set of points $\mathcal{H}_{w}^{m, g} \subset \Gamma$, usually called sparse grid and obtained as a superposition of all full tensor grids appearing in (6.2) that are multiplied by a nonzero coefficient $c(\mathbf{i})$. See Figure 8 for some examples. We finally remark that formula (6.2) is in general not interpolatory unless nested sequences of one-dimensional abscissas are used; see [9].

To fully characterize the sparse approximation operator $\mathcal{S}_{w}^{m, g}$ one has to provide the two strictly increasing functions $m: \mathbb{N}_{+} \rightarrow \mathbb{N}_{+}$and $g: \mathbb{N}_{+}^{N} \rightarrow \mathbb{N}$. The first defines the relation between the level $i$ and the number of points $m(i)$ in the corresponding one-dimensional polynomial interpolation formula $\mathcal{I}^{m(i)}$, while the second characterizes the set of multi-indices used to construct the sparse approximation. The requirement for $g$ to be increasing guarantees that if $\mathbf{i}$ is an admissible multi-index, i.e., $\mathbf{i} \in \Upsilon \equiv\left\{\mathbf{j} \in \mathbb{N}_{+}^{N}, g(\mathbf{j}) \leq w\right\}$, then all multi-indices $\mathbf{j} \leq \mathbf{i}$ are also admissible. Therefore, the index set $\Upsilon$ satisfies the admissibility condition in the sense of [30, section 3.1].

Since $m$ is not surjective in $\mathbb{N}^{+}$(unless it is affine) we introduce a left inverse

$$
m^{-1}(k)=\min \left\{i \in \mathbb{N}_{+}: m(i) \geq k\right\} .
$$

Observe that with this choice $m^{-1}$ is a (nonstrictly) increasing function satisfying $m^{-1}(m(i))=i$ and $m\left(m^{-1}(k)\right) \geq k$.

Let $\mathbf{m}(\mathbf{i})=\left(m\left(i_{1}\right), \ldots, m\left(i_{N}\right)\right)$ and consider the set of polynomial multidegrees

$$
\Lambda^{m, g}(w)=\left\{\mathbf{p} \in \mathbb{N}^{N}, g\left(\mathbf{m}^{-1}(\mathbf{p}+\mathbf{1})\right) \leq w\right\} .
$$


Denote by $\mathbb{P}_{\Lambda^{m, g}(w)}(\Gamma)$ the corresponding multivariate polynomial space spanned by the monomials with multidegree in $\Lambda^{m, g}(w)$, i.e.,

$$
\mathbb{P}_{\Lambda^{m, g}(w)}(\Gamma)=\operatorname{span}\left\{\prod_{n=1}^{N} y_{n}^{p_{n}}, \text { with } \mathbf{p} \in \Lambda^{m, g}(w)\right\} .
$$

The following result proved in [8] states that the sparse approximation formula $\mathcal{S}_{w}^{m, g}$ is exact in $\mathbb{P}_{\Lambda^{m, g}(w)}(\Gamma)$.

Proposition 6.1.

(a) For any $f \in C_{\sigma}^{0}(\Gamma ; V)$, we have $\mathcal{S}_{w}^{m, g}[f] \in \mathbb{P}_{\Lambda^{m, g}(w)} \otimes V$.

(b) Moreover, $\mathcal{S}_{w}^{m, g}[v]=v$ for all $v \in \mathbb{P}_{\Lambda^{m, g}(w)} \otimes V$.

We recall that the most typical choice of $m$ and $g$ is given by (see [53, 9])

$$
m(i)=\left\{\begin{array}{ll}
1 & \text { for } i=1 \\
2^{i-1}+1 & \text { for } i>1
\end{array} \quad \text { and } \quad g(\mathbf{i})=\sum_{n=1}^{N}\left(i_{n}-1\right) .\right.
$$

This choice of $m$, combined with the choice of Clenshaw-Curtis interpolation points (extrema of Chebyshev polynomials), leads to nested sequences of one-dimensional interpolation formulas and a reduced sparse grid.

On the other hand, if we choose $m(i)=i$, it is easy to find functions $g$ for the construction of sparse collocation approximations in standard polynomial spaces, such as tensor product, total degree, etc. Table 1 summarizes several options available.

Table I Sparse approximation formulas and corresponding set of polynomial multidegrees used for

\begin{tabular}{|c|c|c|}
\hline Approx. space & Sparse grid: $\quad m, g$ & Polynomial space: $\quad \Lambda(w)$ \\
\hline Tensor product & $\begin{array}{c}m(i)=i \\
g(\mathbf{i})=\max _{n}\left(i_{n}-1\right) \leq w\end{array}$ & $\left\{\mathbf{p} \in \mathbb{N}^{N}: \max _{n} p_{n} \leq w\right\}$ \\
\hline Total degree & $\begin{array}{c}m(i)=i \\
g(\mathbf{i})=\sum_{n}\left(i_{n}-1\right) \leq w\end{array}$ & $\left\{\mathbf{p} \in \mathbb{N}^{N}: \quad \sum_{n} p_{n} \leq w\right\}$ \\
\hline Hyperbolic cross & $\begin{array}{c}m(i)=i \\
g(\mathbf{i})=\prod_{n}\left(i_{n}\right) \leq w+1\end{array}$ & $\left\{\mathbf{p} \in \mathbb{N}^{N}: \prod_{n}\left(p_{n}+1\right) \leq w+1\right\}$ \\
\hline Smolyak & $\begin{aligned} m(i) & =\left\{\begin{array}{l}2^{i-1}+1, i>1 \\
1, i=1\end{array}\right. \\
g(\mathbf{i}) & =\sum_{n}\left(i_{n}-1\right) \leq w\end{aligned}$ & $\begin{array}{c}\left\{\mathbf{p} \in \mathbb{N}^{N}: \quad \sum_{n} f\left(p_{n}\right) \leq f(w)\right\} \\
f(p)=\left\{\begin{array}{l}0, p=0 \\
1, p=1 \\
\left\lceil\log _{2}(p)\right\rceil, p \geq 2\end{array}\right.\end{array}$ \\
\hline
\end{tabular}
approximation.

It is also straightforward to build related anisotropic sparse approximation formulas by making the function $g$ act differently on the input random variables $y_{n}$. Anisotropic sparse stochastic collocation [45] combines the advantages of isotropic sparse collocation with those of anisotropic full tensor product collocation: the first approach is effective in reducing the curse of dimensionality for problems depending on random variables which weigh approximately equally in the solution, and the second is appropriate for solving highly anisotropic problems with relatively low effective dimension, as in the case where input random variables are Karhunen-Loève truncations of "smooth" random fields. 
This anisotropy is described in terms of properly defined weights, $\boldsymbol{\alpha}=\left(\alpha_{1}, \ldots, \alpha_{N}\right)$. For example, to obtain an anisotropic version of the isotropic Smolyak method in Table 1 we can first define an anisotropic set of indices by the function $g(\mathbf{i} ; \boldsymbol{\alpha})=$ $\sum_{n=1}^{N} \frac{\alpha_{n}}{\alpha_{\min }} i_{n}$, i.e., $\left\{\mathbf{i} \in \mathbb{N}_{+}^{N}: g(\mathbf{i} ; \boldsymbol{\alpha}) \leq w\right\}$, where $\alpha_{\min }=\min _{n} \alpha_{n}$. Then the anisotropic Smolyak approximation formula reads

$$
\mathcal{S}_{w}^{m, g, \boldsymbol{\alpha}}\left[u_{h}\right]=\sum_{\mathbf{i} \in \mathbb{N}_{+}^{N}: g(\mathbf{i} ; \boldsymbol{\alpha}) \leq w} \bigotimes_{n=1}^{N} \Delta_{n}^{m\left(i_{n}\right)}\left(u_{h}\right) .
$$

Anisotropic versions of the other sparse grid approximation formulas presented in Table 1 are detailed in [8]. Figure 8 shows examples of a full tensor, total degree, isotropic Smolyak, and anisotropic Smolyak sparse grid, respectively. Observe that the full tensor and the Smolyak grids both have maximum polynomial degree of approximation in each variable equal to 32 . On the other hand, the total degree sparse grid shown in the figure has only polynomial degree of approximation $p=10$, as the $p=32$ case would result in a grid denser than the tensor product grid, being in this case only two-dimensional.
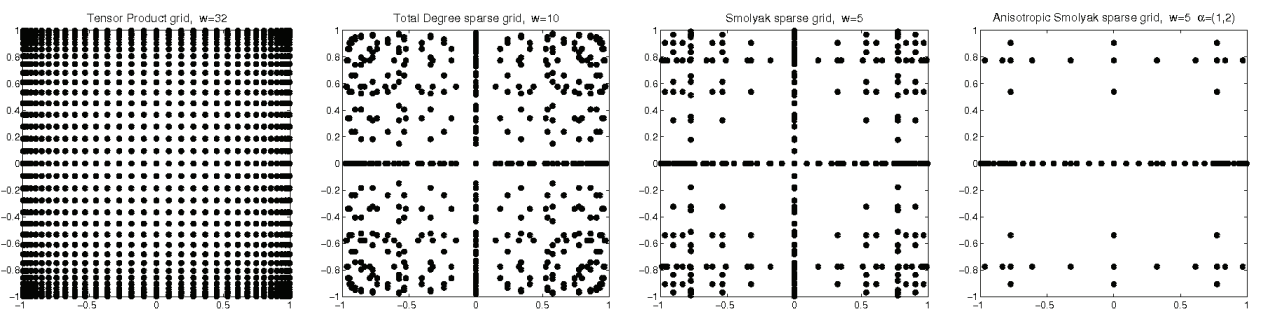

Fig. 8 Examples of grids obtained from formula (6.2). From left to right: tensor product with $w=32$; total degree with $w=10$; Smolyak with $w=5$; anisotropic Smolyak with $w=5$ and $\boldsymbol{\alpha}=(1,2)$.

The key idea in the anisotropic sparse stochastic collocation method is to place more points in those directions with slower convergence rate, tuning the actual number of points to the type of convergence. The actual construction of $\boldsymbol{\alpha}$ can be based on either a priori or a posteriori information, as suggested in [45]. In particular, for problems with analytic dependence on the input random variables the weights $\alpha_{n}$ can be taken equal to the exponential rates, $r_{n}$, introduced in Theorem 4.1, i.e., $\alpha_{n}=r_{n}$, $n=1, \ldots, N$.

In this respect, the methodology described above for tuning the anisotropy to the regularity of the solution differs from the dimension-adaptive method developed in [30] and applied in the context of PDEs with random input data in [29], where the sparse approximation structure has to be uncovered only by successive enrichment, and does not exploit any a priori information on the solution.

A related approach due to Schwab and coworkers (see, for instance, [55]) uses sparse wavelet chaos subspaces. Those approximations are of ANOVA type, are also anisotropic, and use a priori regularity from the exact solution. The main idea in the ANOVA approach is to reduce the curse of dimensionality by approximating the exact solution by a span of tensor products that depends only on at most a given number of input random variables. 
Finally, we mention the possibility of building a piecewise polynomial approximation, also called the multielement probabilistic collocation method, as proposed in [26], where anisotropy is introduced a priori at the level of $h$ discretization.

6.2. Convergence Analysis. Standard techniques for developing error bounds for multidimensional approximations are based on one-dimensional results. This is the reason why the tools developed in section 4 to prove Theorem 4.1 are very useful, even when we no longer use full tensor product approximations. In such a case a general approach is to bound both the discretization error and the number of collocation points $\eta=\eta(w, N)=\# \mathcal{H}_{w}^{m, g}$ in terms of the level $w$. To conclude, one produces a bound of the error in terms of $\eta$, which gives an estimate in the complexity of the method. As examples of this type of result, we state two theorems that apply for bounded input random variables.

The first result, from [46], estimates the convergence rate of the isotropic Smolyak method as defined in Table 1. In this result we focus only on the sparse stochastic collocation discretization error, since the space discretization error can be analyzed exactly as in Theorem 4.1.

THEOREM 6.2. For a function $u \in C^{0}(\Gamma ; V)$ satisfying the assumptions of Theorem 4.1 the isotropic Smolyak formula based on $\hat{\rho}$-Gaussian abscissas satisfies

$$
\begin{aligned}
\left\|u-\mathcal{S}_{w}^{m, g}[u]\right\|_{L_{\rho}^{2}(\Gamma ; V)} & \leq \sqrt{\|\rho / \hat{\rho}\|_{L^{\infty}(\Gamma)}} C\left(r_{\min }, N\right) \eta^{-\mu}, \\
\mu & :=\frac{r_{\min } e \log (2)}{\zeta+\log (N)},
\end{aligned}
$$

with $\zeta:=1+\left(1+\log _{2}(1.5)\right) \log (2) \approx 2.1$. The constant $C\left(r_{\min }, N\right)$ is defined in [46, formula (3.31), p. 2331] and tends to zero as $r_{\min } \rightarrow \infty$.

We recall that an isotropic full tensor product interpolation converges roughly as $C\left(r_{\min }, N\right) \eta^{-r_{\min } / N}$ if $N \gg \log (\eta)$. The slowdown effect that the dimension $N$ has on the last convergence is known as the curse of dimensionality and is the reason for not using isotropic full tensor interpolation for large values of $N$. On the other hand, the isotropic Smolyak approximation seems to be better suited for this case. Indeed, from estimate (6.4) we see that the Smolyak algebraic convergence has the faster exponent $\mathcal{O}\left(\frac{r_{\min }}{\zeta+\log (N)}\right)$. This is a clear advantage of the isotropic Smolyak method with respect to the full tensor and justifies our claim that the use of Smolyak approximation reduces the curse of dimensionality.

The second result we state is taken from [45] and analyzes the rate of convergence for the anisotropic Smolyak formula.

THEOREM 6.3. Let the function $u \in C^{0}(\Gamma ; V)$ satisfy the assumptions of Theorem 4.1. Let the anisotropic Smolyak formula (6.3) be based on $\hat{\rho}$-Gaussian abscissas and anisotropy weights $\alpha_{n}=r_{n}$ with $r_{n}$ as in Theorem 4.1. We have the following estimate:

$$
\begin{aligned}
\left\|u-\mathcal{S}_{w}^{m, g, \mathbf{r}}[u]\right\|_{L_{\rho}^{2}(\Gamma ; V)} & \leq \sqrt{\|\rho / \hat{\rho}\|_{L^{\infty}(\Gamma)}} \hat{C}(\mathbf{r}, N) \eta^{-\tilde{\mu}}, \\
\tilde{\mu} & :=\frac{r_{\min } \log (2) e}{2 \log (2)+\sum_{n=1}^{N} r_{\min } / r_{n}},
\end{aligned}
$$

with the constant $\hat{C}(\mathbf{r}, N)$ depending only on the rate vector $\mathbf{r}=\left(r_{1}, \ldots, r_{N}\right)$; see $[45$, Theorem 3.13]. 
The remarkable property of estimate (6.5) is that the exponent $\tilde{\mu}$ depends on the dimension $N$ only through the term $\sum_{n=1}^{N} r_{\min } / r_{n}$. Suppose now that the stochastic input data are truncated expansions of random fields and that we are able to estimate the values $\left\{r_{n}\right\}_{n=1}^{\infty}$. Whenever the sum $\sum_{n=1}^{\infty} 1 / r_{n}$ is finite, then the algebraic exponent in (6.5) does not deteriorate as the truncation dimension $N$ increases. It is also shown in [45] that, under the same condition, the constant $\hat{C}(\mathbf{r}, N)$ is uniformly bounded with respect to $N$. This condition is satisfied, for instance, by the problem considered in section 6.4.1. This is a clear advantage with respect to the isotropic Smolyak method since, in this case, the anisotropic method does not suffer from the curse of dimensionality. In fact, in such a case we can work directly with the anisotropic Smolyak formula (6.3) in infinite dimensions: here the vector of indices becomes natural sequences, and the constraint that defines the index set is $\sum_{n=1}^{\infty}\left(i_{n}-1\right) r_{n} \leq w r_{\min }$.

Similar results hold for other PDEs with analytic dependence on the input variables; see, for instance, [44]. Furthermore, an error estimate for unbounded random variables with Gaussian tails can be obtained in a completely analogous way using the results of this work, in particular Lemma 4.6.

For ANOVA-type sparse approximation the analysis has been carried out in [55].

6.3. More on Regularity Results and Convergence Rates. As we have seen in the previous section, to build an effective anisotropic sparse grid approximation one needs a sharp estimate of the exponential convergence rates $r_{n}$ for one-dimensional interpolation. This actually turns back to studying the regularity of the solution $u: \Gamma \rightarrow H_{0}^{1}(D)$ of the parametric problem (1.7) with respect to each variable $y_{n}$, i.e., looking at the function $u\left(y_{n}, y_{n}^{*}, x\right)$ as a function of $y_{n}, u: \Gamma_{n} \rightarrow C_{\sigma_{n}^{*}}^{0}\left(\Gamma_{n}^{*} ; H_{0}^{1}(D)\right)$.

This was done in section 3 ; in particular, Lemma 3.2 characterizes the analyticity region of the function $u(z)=u\left(z, y_{n}^{*}, x\right), z \in \mathbb{C}$, by checking the radius of convergence of the Taylor series in every point $y_{n} \in \Gamma_{n}$.

Another procedure that sometimes leads to a sharper characterization of the analyticity region consists in studying directly problem (1.7) in the complex domain, i.e., by replacing $y_{n}$ with a complex variable $z \in \mathbb{C}$ and looking for a complex solution $u: \mathbb{C} \rightarrow C_{\sigma_{n}^{*}}^{0}\left(\Gamma_{n}^{*} ; H_{0}^{1}(D)\right)$. The analyticity region $\Sigma\left(\Gamma_{n}, \tau_{n}\right)$ is then characterized by those values $z \in \mathbb{C}$ for which the complex problem is well posed and the solution $u(z)$ satisfies the Cauchy-Riemann conditions. This approach is detailed in [44].

We give some details for the linear problem (1.7) with a deterministic right-hand side $f$. With the notation $a(z)=a\left(z, y_{n}^{*}, x\right)$, the following lemma can be shown [44].

Lemma 6.4. The solution $u: \mathbb{C} \rightarrow C_{\sigma_{n}^{*}}^{0}\left(\Gamma_{n}^{*} ; H_{0}^{1}(D)\right)$ is analytic in the region of the complex plane $\Sigma_{n} \equiv\{z \in \mathbb{C}: \operatorname{Re}(a(z))>0$, a $(z)$ analytic in $z\}$.

If we consider a linear truncated Karhunen-Loève expansion as in Example 3 and assume that the random variables are bounded, i.e., $\Gamma_{n}=\left(y_{n}^{\min }, y_{n}^{\max }\right)$, then the analyticity region can be estimated as

$$
\Sigma_{n} \equiv\left\{z \in \mathbb{C}: \quad y_{n}^{\min }-\tau_{n} \leq \operatorname{Re}(z)<y_{n}^{\max }+\tau_{n}\right\}, \quad \text { with } \tau_{n}=\frac{a_{\min }}{2 \sqrt{\lambda_{n}}\left\|b_{n}\right\|_{L^{\infty}(D)}},
$$

which is larger than the one given in section 3 and, in particular, has no limitation on $\operatorname{Im}(z)$.

Introducing now the scaling $z(t)=\bar{y}_{n}+\frac{\left|\Gamma_{n}\right|}{2} t, t \in \mathbb{C}$, with $\bar{y}_{n}=\frac{y_{n}^{\max }+y_{n}^{\min }}{2}$, the scaled function $\tilde{u}(t)=u(z(t))$ is analytic in the region $\tilde{\Sigma}_{n}=\{t \in \mathbb{C}:|\operatorname{Re}(t)| \leq$ $\left.1+\frac{2 \tau_{n}}{\left|\Gamma_{n}\right|}\right\}$. Therefore, following the argument in Lemma 4.4, we see that the largest Bernstein ellipse $E_{\rho}$ (4.3) that can be drawn in the region $\tilde{\Sigma}_{n}$ has larger semi-axis 
$\left(\varrho+\varrho^{-1}\right) / 2=1+\tau_{n}$, and the exponential convergence rate in Theorem 4.1 can be improved to

$$
r_{n}=\log \left[1+\frac{2 \tau_{n}}{\left|\Gamma_{n}\right|}\left(1+\sqrt{1+\frac{\left|\Gamma_{n}\right|}{\tau_{n}}}\right)\right] .
$$

This estimate has been used in [8] to construct better anisotropic polynomial approximations (see section 6.4.2).

6.4. Numerical Examples. Here we consider two numerical examples for the diffusion problem in the form of (1.1) defined in the unit square $[0,1]^{2}$, with homogeneous Dirichlet boundary conditions and a stochastic conductivity coefficient that depends on a finite number of random variables. The first example shows the effectiveness of anisotropic stochastic collocation for a problem with a large number of input random variables. The second provides a comparison with stochastic Galerkin in terms of computational efficiency.

6.4.I. First Example. This numerical example is taken from [45]. It uses a deterministic load $f(\omega, x, z)=\cos (x) \sin (z)$ and a random diffusion coefficient $a_{N}(\omega, x)$ representing a stratified medium, given by

$$
\log \left(a_{N}(\omega, x)-0.5\right)=1+\mathrm{Y}_{1}(\omega)\left(\frac{\sqrt{\pi} L_{c}}{2}\right)^{1 / 2}+\sum_{n=2}^{N} \zeta_{n} \varphi_{n}(x) \mathrm{Y}_{n}(\omega),
$$

where the weights $\zeta_{n}$ have the decay

$$
\zeta_{n}:=\left(\sqrt{\pi} L_{c}\right)^{1 / 2} \exp \left(\frac{-\left(\left\lfloor\frac{n}{2}\right\rfloor \pi L_{c}\right)^{2}}{8}\right) \quad \text { if } n>1
$$

and the functions $\varphi_{n}(x)$ are taken as

$$
\varphi_{n}(x):= \begin{cases}\sin \left(\left\lfloor\frac{n}{2}\right\rfloor \pi x\right) & \text { if } n \text { even, } \\ \cos \left(\left\lfloor\frac{n}{2}\right\rfloor \pi x\right) & \text { if } n \text { odd. }\end{cases}
$$

Here $L_{c}$ represents a physical correlation length for the random field $a$, meaning that the random variables $a\left(x_{1}\right)$ and $a\left(x_{2}\right)$ become essentially uncorrelated for $\mid x_{1}-$ $x_{2} \mid \gg L_{c}$. Expression (6.7) represents a possible truncation of a one-dimensional random field with stationary covariance

$$
\operatorname{cov}\left[\log \left(a_{N}-0.5\right)\right]\left(x_{1}, x_{2}\right)=\exp \left\{-\frac{\left(x_{1}-x_{2}\right)^{2}}{L_{c}^{2}}\right\} .
$$

In this example, the random variables $\left\{Y_{n}(\omega)\right\}_{n=1}^{N}$ are independent, have zero mean and unit variance, i.e., $\mathbb{E}\left[Y_{n}\right]=0$ and $\mathbb{E}\left[Y_{n} Y_{m}\right]=\delta_{n m}$ for $n, m \in \mathbb{N}_{+}$, and are uniformly distributed in the interval $[-\sqrt{3}, \sqrt{3}]$. The correlation length is $L_{c}=1 / 16$.

Figure 9 shows the convergence rate for the anisotropic Smolyak method, for various levels of truncation in the expansion (6.7). The anisotropy weights $\alpha_{n}$ have been computed a posteriori performing a one-dimensional numerical convergence analysis (see [45, section 2.2] for details).

Observe the remarkable performance of the anisotropic Smolyak method. The convergence rate is dimension dependent, but due to our algorithm's inherent capability to adapt itself to the eigenvalue decay of (6.7), we notice that this dependence 


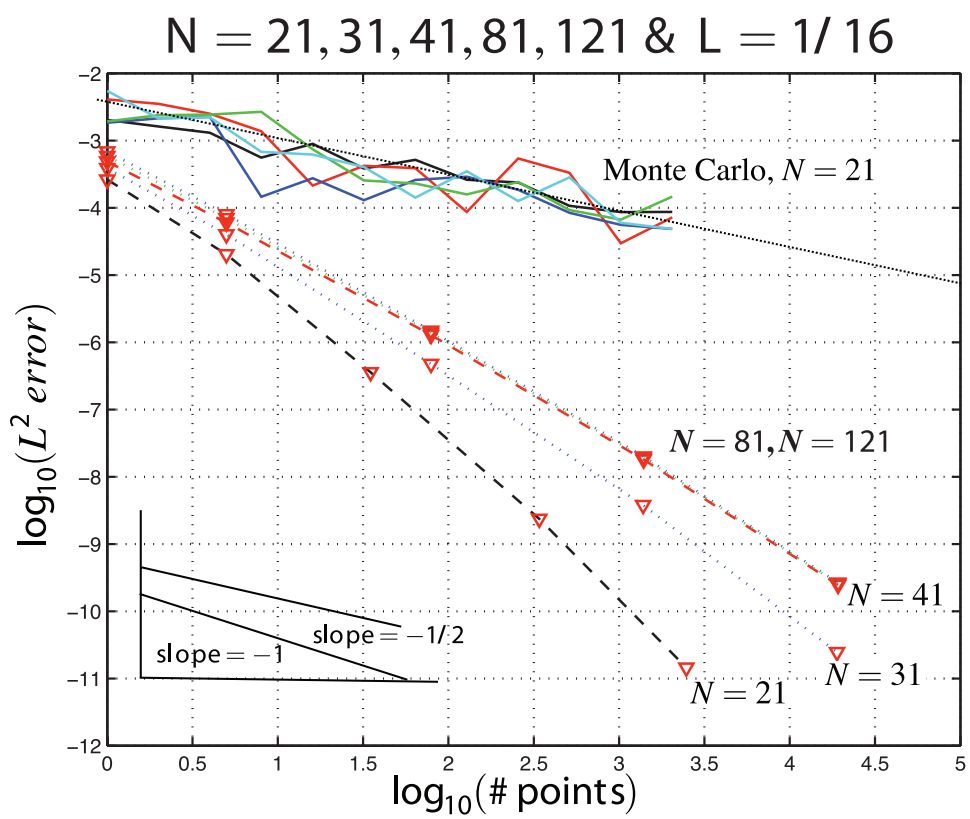

- - - - - Anisotropic Smolyak with Clenshaw-Curtis abscissas ( $N=21)$

-- - - - Anisotropic Smolyak with Clenshaw-Curtis abscissas ( $N=31$ )

- - - - Anisotropic Smolyak with Clenshaw-Curtis abscissas ( $N=41)$

-- - - - Anisotropic Smolyak with Clenshaw-Curtis abscissas $(\mathrm{N}=81)$

.......... Anisotropic Smolyak with Clenshaw-Curtis abscissas $(\mathrm{N}=121)$

Fig. 9 A 21-, 31-, 41-, 81-, 121-dimensional comparison of the anisotropic Smolyak method, with a posteriori selection of the $\boldsymbol{\alpha}$ weights, and a 21-dimensional Monte Carlo approach for solving problem (1.1) with diffusion coefficient (6.7) and correlation length $L_{c}=1 / 16$. We plot the $L^{2}(D)$ approximation error in the expected value versus the number of collocation (sample) points.

is no longer evident as we increase the dimension from $N=41$ to $N=81$ and further to $N=121$. Furthermore, the dominance of the anisotropic sparse grid method is further displayed when comparing its convergence in 121 stochastic dimensions to that of Monte Carlo in only 21 dimensions.

6.4.2. Second Example. This example, taken from [8], represents a thermal conduction problem in a medium with four circular inclusions with random conductivity (see Figure 10, left). The problem is as in (1.1) with homogeneous Dirichlet boundary conditions and a heat source uniformly distributed on the whole domain. The random coefficient is $a(\omega, \mathbf{x})=1+\sum_{n=1}^{4} \gamma_{n} y_{n}(\omega) \chi_{n}(\mathbf{x})$, where $\chi_{n}$ is the indicator function for each circle, $Y_{n}(\omega) \sim \mathcal{U}(-0.99,0)$, and $\gamma_{n} \leq 1$. The values of the coefficients $\gamma_{n}$ are shown in Figure 10, left. Notice that these values give different importance to the four random variables. In particular, the inclusion in the bottom-left corner has the largest variance, and we expect it to contribute the most to the total variance of the solution. It is therefore intuitively justified to use polynomial degrees higher in the corresponding direction of the stochastic multidimensional space rather than in the other ones.

We compare the accuracy of the stochastic Galerkin and stochastic collocation methods by looking at statistical indicators of the following quantity of interest: 

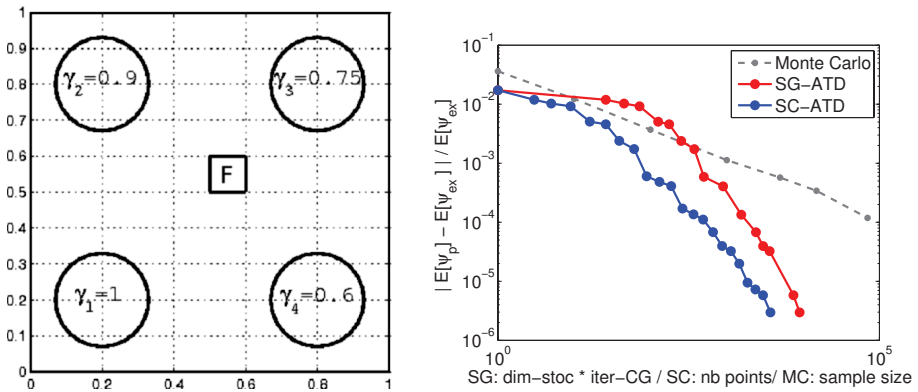

Fig. 10 Comparison between $S G$-ATD and $S C$-ATD methods with theoretical weights in the computation of $\mathbb{E}[\psi]$. Error $\varepsilon_{\text {mean }}[\psi]$ versus computational cost.

$\psi(u)=\int_{F} u(\mathbf{x}) d \mathbf{x}$. Let $u_{p}$ be an approximate solution (computed either with stochastic Galerkin or stochastic collocation) and $u_{e x}$ the exact solution. We check here the convergence of the following error in the mean: $\varepsilon_{\text {mean }}[\psi]=\left|\mathbb{E}\left[\psi\left(u_{p}\right)\right]-\mathbb{E}\left[\psi\left(u_{e x}\right)\right]\right|$.

In Figure 10, right, we compare the performances of the anisotropic total degree stochastic Galerkin (SG-ATD) method and the anisotropic total degree sparse grid method (SC-ATD) using the theoretical weights $\alpha_{n}=r_{n}$ estimated from (6.6) and Example 3 . The horizontal axis shows the estimated computational cost that, for the collocation method, is given in terms of the number of collocation points in the sparse grid and, for the Galerkin method, in terms of the dimension of the anisotropic total degree polynomial space multiplied by the number of block diagonal preconditioned conjugate gradient iterations to solve the coupled linear system. In this test, the collocation method seems to be superior to the Galerkin one, even for very small tolerances.

7. Conclusions. The original version of this revised article [7] proposed a collocation method for the solution of elliptic PDEs with random coefficients and forcing terms. This method requires the solution of fully parallelizable, uncoupled deterministic problems also in the case of input data which depend nonlinearly on the random variables; treats efficiently the case of nonindependent random variables with the introduction of an auxiliary density $\hat{\rho}$; deals easily with unbounded random variables, such as Gaussian or exponential ones; and deals with no difficulty with a diffusivity coefficient $a$ with unbounded second moment.

The work [7] provided a full convergence analysis and proved exponential convergence "in probability" for a broad range of situations. The theoretical result is given in Theorem 4.1 and confirmed numerically by the tests presented in section 5 .

The extension of the analysis to other classes of linear and nonlinear problems is the subject of ongoing research.

The use of tensor product polynomials, even anisotropic ones as suggested in [7], suffers from the curse of dimensionality. Hence, this method is efficient only for a small number of random variables or, equivalently, for a small effective dimension. For moderate or large dimensionality of the probability space, one should rather turn to sparse tensor product spaces. This aspect was investigated in subsequent works.

The work [46] analyzed a sparse grid stochastic collocation method for solving PDEs whose coefficients and forcing terms depend on a finite number of random variables. The sparse grids were constructed from the Smolyak formula, utilizing either Clenshaw-Curtis or Gaussian abscissas. 
Then the work [45] proposed and analyzed a novel weighted Smolyak method, describing an optimal choice for the weight parameters. These weights tune the anisotropy of the approximation to each given problem. Their systematic choice can be based either on a priori or a posteriori information and is motivated by the regularity of the solution and the error estimates derived in [45].

In fact, given a PDE with random data, a crucial problem is to find optimal approximation spaces. This interesting problem appears not only in the realm of stochastic collocation. In the area of stochastic Galerkin there has been active research to provide better types of approximation including $h-p$ type, also called the multielement method $[4,58]$ and ANOVA-type expansions as in [55].

On the other hand the works [22, 47] (see also the references therein) have pursued generalized spectral decompositions for approximation, seeking reduced bases that are tailored to the solution and which seem to be rather promising. Approximation of low regularity functions in high dimensions is particularly difficult; see, for instance, $[38,37,41,16,17]$. It is worth mentioning that the development of numerical methods for PDEs with random coefficients is still very much ongoing, and better numerical methods are expected to appear.

REFERENCES

[1] M. Ainsworth And J.-T. Oden, A Posteriori Error Estimation in Finite Element Analysis, Wiley, New York, 2000.

[2] I. BABušKA, K.-M. Liu, And R. Tempone, Solving stochastic partial differential equations based on the experimental data, Math. Models Methods Appl. Sci., 13 (2003), pp. 415-444.

[3] I. BabušKa And T. Strouboulis, The Finite Element Method and Its Reliability, Numerical Mathematics and Scientific Computation, Clarendon Press, Oxford University Press, New York, 2001.

[4] I. BABušKa, R. Tempone, And G. E. Zouraris, Galerkin finite element approximations of stochastic elliptic partial differential equations, SIAM J. Numer. Anal., 42 (2004), pp. 800825 .

[5] I. BabušKa, R. Tempone, And G. E. Zouraris, Solving elliptic boundary value problems with uncertain coefficients by the finite element method: The stochastic formulation, Comput. Methods Appl. Mech. Engrg., 194 (2005), pp. 1251-1294.

[6] I. Babuška, F. Nobile, and R. Tempone, Worst-case scenario analysis for elliptic problems with uncertainty, Numer. Math., 101 (2005), pp. 185-219.

[7] I. BabušKa, F. Nobile, and R. Tempone, A stochastic collocation method for elliptic partial differential equations with random input data, SIAM J. Numer. Anal., 45 (2007), pp. 10051034.

[8] J. BÄck, F. Nobile, L. Tamellini, And R. Tempone, Stochastic spectral Galerkin and collocation methods for PDEs with random coefficients: A numerical comparison, in Proceedings of the International Conference on Spectral and High Order Methods (ICOSAHOM 09), Springer-Verlag, Berlin, 2010.

[9] V. Barthelmann, E. Novak, And K. Ritter, High dimensional polynomial interpolation on sparse grids, Adv. Comput. Math., 12 (2000), pp. 273-288.

[10] M. Bieri, R. Andreev, and C. Schwab, Sparse Tensor Discretization of Elliptic sPDEs, SAM Report 2009-07, Seminar für Angewandte Mathematik, ETH, Zurich, 2009.

[11] J. P. Boyd, Asymptotic coefficients of Hermite function series, J. Comput. Phys., 54 (1984), pp. $382-410$.

[12] J. P. Boyd, Chebyshev and Fourier Spectral Methods, 2nd ed., Dover, Mineola, NY, 2001.

[13] M. BraAcK And A. ERn, A posteriori control of modeling errors and discretization errors, Multiscale Model. Simul., 1 (2003), pp. 221-238.

[14] S. C. Brenner And L. R. Scott, The Mathematical Theory of Finite Element Methods, Springer-Verlag, New York, 1994.

[15] H.-J. Bungartz And M. Griebel, Sparse grids, Acta Numer., 13 (2004), pp. 147-269.

[16] C. Canuto And D. Fransos, Numerical solution of partial differential equations in random domains: An application to wind engineering, Commun. Comput. Phys., 5 (2009), pp. 515531. 
[17] T. Chantrasmi, A. Doostan, and G. Iaccarino, Padé-Legendre approximants for uncertainty analysis with discontinuous response surfaces, J. Comput. Phys., 228 (2009), pp. $7159-7180$.

[18] P. G. Ciarlet, The Finite Element Method for Elliptic Problems, North-Holland, New York, 1978.

[19] A. Cohen, R. DeVore, and C. Schwab, Convergence Rates of Best N-term Galerkin Approximations for a Class of Elliptic sPDEs, SAM Report 2009-02, Seminar für Angewandte Mathematik, ETH, Zurich, 2009.

[20] M. K. Deb, I. BabušKa, And J. T. Oden, Solution of stochastic partial differential equations using Galerkin finite element techniques, Comput. Methods Appl. Mech. Engrg., 190 (2001), pp. 6359-6372.

[21] R. A. DeVore And G. G. Lorentz, Constructive Approximation, Grundlehren Math. Wiss. 303, Springer-Verlag, Berlin, 1993.

[22] A. Doostan, R. G. Ghanem, And J. Red-Horse, Stochastic model reduction for chaos representations, Comput. Methods Appl. Mech. Engrg., 196 (2007), pp. 3951-3966.

[23] H. C. Elman, O. G. Ernst, D. P. O'Leary, and M. Stewart, Efficient iterative algorithms for the stochastic finite element method with application to acoustic scattering, Comput. Methods Appl. Mech. Engrg., 194 (2005), pp. 1037-1055.

[24] B. Engquist, P. Löstedt, And O. Runborg, Eds., Multiscale Methods in Science and Engineering, Lect. Notes Comput. Sci. Eng. 44, Springer-Verlag, Berlin, 2005.

[25] P. ERdös AND P. TurÁn, On interpolation. I. Quadrature- and mean-convergence in the Lagrange-interpolation, Ann. of Math. (2), 38 (1937), pp. 142-155.

[26] J. Foo, X. WAn, AND G. E. KARniadakis, The multi-element probabilistic collocation method (ME-PCM): Error analysis and applications, J. Comput. Phys., 227 (2008), pp. 9572-9595.

[27] P. Frauenfelder, C. Schwab, and R. A. Todor, Finite elements for elliptic problems with stochastic coefficients, Comput. Methods Appl. Mech. Engrg., 194 (2005), pp. 205228.

[28] D. Funaro and O. Kavian, Approximation of some diffusion evolution equations in unbounded domains by Hermite functions, Math. Comp., 57 (1991), pp. 597-619.

[29] B. Ganapathysubramanian and N. Zabaras, Sparse grid collocation schemes for stochastic natural convection problems, J. Comput. Phys., 225 (2007), pp. 652-685.

[30] T. Gerstner and M. Griebel, Dimension-adaptive tensor-product quadrature, Computing, 71 (2003), pp. 65-87.

[31] R. GHanem, Ingredients for a general purpose stochastic finite elements implementation, Comput. Methods Appl. Mech. Engrg., 168 (1999), pp. 19-34.

[32] R. G. Ghanem and P. D. Spanos, Stochastic Finite Elements: A Spectral Approach, SpringerVerlag, New York, 1991.

[33] M. Grigoriu, Stochastic Calculus: Applications in Science and Engineering, Birkhäuser Boston, Boston, 2002.

[34] E. Hille, Contributions to the theory of Hermitian series. II. The representation problem, Trans. Amer. Math. Soc., 47 (1940), pp. 80-94.

[35] J. Hlaváček, I. Chleboun, And I. BabušKa, Uncertain Input Data Problems and the Worst Scenario Method, Elsevier, Amsterdam, 2004.

[36] S. Larsen, Numerical Analysis of Elliptic Partial Differential Equations with Stochastic Input Data, Ph.D. thesis, University of Maryland, College Park, MD, 1986.

[37] O. P. Le Maître, O. M. Knio, H. N. Najm, and R. G. Ghanem, Uncertainty propagation using Wiener-Haar expansions, J. Comput. Phys., 197 (2004), pp. 28-57.

[38] O. P. Le Maître, H. N. Najm, P. P. Pébay, R. G. Ghanem, and O. M. Knio, Multiresolution-analysis scheme for uncertainty quantification in chemical systems, SIAM J. Sci. Comput., 29 (2007), pp. 864-889.

[39] M. LoÈve, Probability Theory. I, 4th ed., Grad. Texts in Math. 45, Springer-Verlag, New York, 1977.

[40] M. LoÈve, Probability Theory. II, 4th ed., Grad. Texts in Math. 46, Springer-Verlag, New York, 1978.

[41] X. Ma And N. Zabaras, An adaptive hierarchical sparse grid collocation algorithm for the solution of stochastic differential equations, J. Comput. Phys., 228 (2009), pp. 3084-3113.

[42] L. Mathelin, M. Y. Hussaini, And T. A. ZANG, Stochastic approaches to uncertainty quantification in CFD simulations, Numer. Algorithms, 38 (2005), pp. 209-236.

[43] H. G. Matthies And A. Keese, Galerkin methods for linear and nonlinear elliptic stochastic partial differential equations, Comput. Methods Appl. Mech. Engrg., 194 (2005), pp. 12951331. 
[44] F. Nobile And R. Tempone, Analysis and implementation issues for the numerical approximation of parabolic equations with random coefficients, Internat. J. Numer. Methods Engrg., 80 (2009), pp. 979-1006.

[45] F. Nobile, R. Tempone, and C. G. Webster, An anisotropic sparse grid stochastic collocation method for partial differential equations with random input data, SIAM J. Numer. Anal., 46 (2008), pp. 2411-2442.

[46] F. Nobile, R. Tempone, and C. G. Webster, A sparse grid stochastic collocation method for partial differential equations with random input data, SIAM J. Numer. Anal., 46 (2008), pp. 2309-2345.

[47] A. NouY, Generalized spectral decomposition method for solving stochastic finite element equations: Invariant subspace problem and dedicated algorithms, Comput. Methods Appl. Mech. Engrg., 197 (2008), pp. 4718-4736.

[48] E. NovaK AND K. RitTer, High dimensional integration of smooth functions over cubes, Numer. Math., 75 (1996), pp. 79-97.

[49] J. T. Oden and S. Prudhomme, Estimation of modeling error in computational mechanics, J. Comput. Phys., 182 (2002), pp. 496-515.

[50] J. T. Oden And K. S. Vemaganti, Estimation of local modeling error and goal-oriented adaptive modeling of heterogeneous materials. I. Error estimates and adaptive algorithms, J. Comput. Phys., 164 (2000), pp. 22-47.

[51] A. Quarteroni and A. Valli, Numerical Approximation of Partial Differential Equations, Springer Ser. Comput. Math. 23, Springer-Verlag, Berlin, 1994.

[52] L. J. Roman And M. SARKIS, Stochastic Galerkin method for elliptic SPDEs: A white noise approach, Discrete Contin. Dyn. Syst. Ser. B, 6 (2006), pp. 941-955.

[53] S. A. Smolyak, Quadrature and interpolation formulas for tensor products of certain classes of functions, Dokl. Akad. Nauk SSSR, 4 (1963), pp. 240-243.

[54] M. A. TATANG, Direct Incorporation of Uncertainty in Chemical and Environmental Engineering Systems, Ph.D. thesis, MIT, Cambridge, MA, 1995.

[55] R. A. Todor And C. SchwaB, Convergence rates for sparse chaos approximations of elliptic problems with stochastic coefficients, IMA J. Numer. Anal., 27 (2007), pp. 232-261.

[56] J. V. Uspensky, On the convergence of quadrature formulas related to an infinite interval, Trans. Amer. Math. Soc., 30 (1928), pp. 542-559.

[57] R. Verfürth, A Review of A Posteriori Error Estimation and Adaptive Mesh Refinement Techniques, Teubner, Wiley, Stuttgart, 1996.

[58] X. WAn AND G. E. Karniadakis, Multi-element generalized polynomial chaos for arbitrary probability measures, SIAM J. Sci. Comput., 28 (2006), pp. 901-928.

[59] N. Wiener, The homogeneous chaos, Amer. J. Math., 60 (1938), pp. 897-936.

[60] C. L. Winter AND D. M. TARTAKOvSKy, Groundwater flow in heterogeneous composite aquifers, Water Resour. Res., 38 (2002), article 1148.

[61] D. XIU AND J. S. Hesthaven, High-order collocation methods for differential equations with random inputs, SIAM J. Sci. Comput., 27 (2005), pp. 1118-1139.

[62] D. Xiu And G. E. Karniadakis, Modeling uncertainty in steady state diffusion problems via generalized polynomial chaos, Comput. Methods Appl. Mech. Engrg., 191 (2002), pp. 49274948.

[63] D. Xiu and G. E. Karniadakis, The Wiener-Askey polynomial chaos for stochastic differential equations, SIAM J. Sci. Comput., 24 (2002), pp. 619-644.

Copyright (c) by SIAM. Unauthorized reproduction of this article is prohibited. 